\title{
WAVE TRANSMISSION OVER NON-UNIFORM CABLES AND LONG-DISTANCE AIR-LINES.
}

\author{
BY M. I. PUPIN, PH.D.
}

This paper describes an experimental investigation of a method of constructing cables and long-distance air-lines for power transmission by electrical waves, particularly for long-distance telephony and telegraphy. This method is a practical application which offers an experimental test of the general mathematical theory of wave-propagation over non-uniform conductors, which is given in the second part of the paper. The first part contains a physical explanation of this mathematical theory and a description of experimental researches bearing upon it. It also describes an experimental verification of long-distance telephony over non uniform cables.

\section{Physical Theory of Electrical Wave-Propagation over} Cables and Long-Distance Air-Lines.

A. Wave Propagation Over Uniform Conductors.-Transmission of electrical energy over conducting wires is a wave transmission when the distance between the transmitting and the receiving apparatus is sufficiently long to permit the development of electrical waves. Such a transmission exists in longdistance telegraphy and telephony. It does not exist to any practically appreciable extent in ordinary transmissions of electrical power by alternating currents over distances which up to the present time have been bridged over by electrical power transmission lines. In cases of wave transmission considered here the conductors will be called wave conductors. The circumstances attending wave transmission are considerably different from those attending ordinary electrical transmission and should be carefully differentiated from them. In ordinary transmission 
the reactions set up in the receiving apparatus are the most essential reactions which the force impressed by the transmitting generator has to overcome. The reactions set up in the transmitting line itself are small in comparison to it. The case is analogous to the transmission of power from the piston of a steam engine to a motor connected to the engine by a short, stiff, piston rod. The reactions set up in the piston rod itself are small in comparison to the reactions which the motor opposes to the driving pressure. Hence, neither the elastic nor the kinetic reactions of the piston rod, nor the reactions due to internal frictional resistances in the rod are seriously thought of when we analyze the reactions attending this case of power transmission. But consider now what will happen if we increase the distance between the piston and the receiving motor and consequently increase the length of the piston rod. We can no longer consider the rod as a perfectly rigid connection between the driving pressure of the piston and the reactions of the receiving motor. The rate at which the piston delivers energy at any moment is not equal to the rate at which energy is delivered at the receiving motor at that moment. There is a lag in phase. The energy transmitted is first stored up in the piston rod and then delivered from the rod to the receiving motor. While it is stored up in the rod it exists partly as kinetic energy of the moving mass of the rod and partly as potential energy, due to the rod's elastic deformations. The process of transmission consists in successive transformations of the kinetic into the potential energy of the rod and vice versa. These transformations being progressive the energy is propagated along the rod, and we say that the propagation is a wave propagation, in order to state in a single word that the progressive motion along the rod is a periodic one. Analogous conditions exist when electrical energy is transmitted by a periodically varying electromotive force acting on a long conductor. The transmission is not a direct one; the transmitted energy is first stored up in the medium surrounding the transmission line, and from there it is transferred to the receiving apparatus. While it is stored up in the medium it exists there partly as mag netic energy stored up in the field of magnetic flux and partly as electrical energy stored up in the field of electrical flux. The process of propagation consists in the progressive transformation of the magnetic into the electrical energy and vice versa. When the electromotive force impressed by the transmitting generator is a peri- 
odic one the propagation will be in the form of electrical waves. The expression "electrical wave" is nothing more nor less than a brief statement of the physical fact that in the case under consideration the energy which at any moment is stored up in the medium surrounding the transmission line is distributed periodically over this line. The current and the potential also vary periodically. At points of maximum magnetic energy the current is maximum, and at points of maximum electrical energy the potential is maximum. Roughly speaking, points of maximum current are points of minimum potential and vice versa.

Wave-length.-Consider now the distance between any two consecutive points of minimum current or minimum potential. This distance is a half wave-length. Suppose that the impressed electromotive force is a simple harmonic of frequency 600 p.p.s. Say that we find the wave-length to be 18 miles, the velocity of propagation will be 10,800 miles per second; considerably less than the velocity of propagation of light through a vacuum. To some this numerical illustration may seem as highly improbable, for we are accustomed to hear much of electricity being propagated with the velocity of light. But it should be remembered that this is true under certain particular conditions only. The velocity of propagation of electrical waves of telephonic frequencies over conducting wires may be anything from the velocity of light down to a few inches, or even less than an inch, per second, depending on the inductance, resistance, and capacity of the line. The smaller the velocity the shorter, of course, will be the wave-length for a given frequency. The wave-length, as will be seen presently, plays a very important part in this investigation. It is considered here as one of the characteristic constants of wave propagation. I ain not aware that previous investigators of the propagation of long electrical waves have devoted any serious attention to this characteristic constant.

Attenuation Constant.-There still remains another constant which with the wave-length completely defines electrical wave propagation. It is called here the attenuation constant. To bring out its physical meaning consider two consecutive half wave-lengths at any moment. The one nearest to the transmitting apparatus shall be denoted by $\mathrm{A}$ and the other by в. The wave energy stored up in the medium surrounding $\mathrm{A}$ is greater than that stored up in the medium surrounding B. Hence wave energy is gradually dissipated during its propagation from the 
transmitting to the receiving apparatus, and therefore the amplitude of both current and potential become smaller as the energy progresses along the transmission line. Let $U$ be the amplitude of the current at the transmitting end, and $U_{\mathrm{s}}$ be the amplitude at a distance $\mathrm{s}$, then if the line be considered infinitely long

$$
\frac{U_{\mathrm{B}}}{U}=e^{-\beta s}
$$

where $e$ is the base of Naperian logarithms. The constant $\beta$ is called here the attenuation constant. The mathematical expression for $\beta$ is well known

$$
\beta=\sqrt{\frac{1}{2} p C\left[\sqrt{p^{2} L^{2}+R^{2}}-p L\right]}
$$

where $L, R, C$, are the inductance, resistance, and capacity, respectively, of the wave conductor per unit length, and $p$ is the frequency speed. Much confusion exists in the minds of physicists as to the real significance of this constant, and as to the true cause of current attenuation. It is usually stated that the capacity of the line, acting as it does somewhat like a shunt, is the cause of all the trouble experienced in electrical wave transmission. This statement contains a small part of the truth, only, and for that reason may and actually has become misleading. The fact that a conductor possesses inductance and capacity shows that the medium surrounding it is capable of storing up energy, which, indeed, is a blessing; it cannot possibly signify that energy propagated along it will be dissipated; and if capacity cannot cause a loss of energy, how can it possibly cause an attenuation of current? The dissipation is due to imperfect conductivity of the wire, and to that alone. Inductance and capacity regulate it, they do not cause it.

Consider now the manner in which this regulation is effected. The dissipation of the energy transmitted occurs at the time when it is stored up in the medium as magnetic energy; for if the medium surrounding an element $d s$ of the transmission wire contains a quantity $d$. $W$ of magnetic energy, then a current $x$ must flow in that element such that

$$
d W=\frac{1}{2} L x^{2} d s
$$


Let $d H$ be the rate of dissipation in that element, then

$$
d H=R x^{2} d s
$$

Suppose now that by some means we increase $L$ to $n^{2} L$, the medium surrounding the elements $d s$ will store up the same amount $d W$ of magnetic with one $n^{\text {th }}$ of the current, for

$$
d W=\frac{1}{2} n^{2} L(x / n)^{2} d s
$$

Let $d H_{1}$ be the rate of dissipation in this case then

$$
d H_{1}=R(x / n)^{2} d s=d H / n^{2}
$$

It follows, therefore, that during the transmission of a given quantity of energy over a conducting wire the dissipation will be diminished by increasing the inductance of the wire, for if the wire have high inductance then small currents are required to transmit a given quantity of energy, and small currents incur small ohmic resistance losses.

By increasing the inductance the efficiency of transmission is increased just as effectively as by increasing the conductivity of the transmission wire.

Distortionless Wave Conductors.-Another.important advantage is gained by increasing the inductance. The expression for $\beta$ given above shows that attenuation depends on frequency; it increases with it. Hence, in telephonic transmission where waves of complex harmonic frequencies are propagated over the line there will be distortion of the waves, because upper harmonics will be attenuated more vigorously than the lower frequencies. This results in a distortion of speech, which is noticed in long. distance telephonic transmission as defective articulation. Some instructive experiments bearing upon this point will be described presently. High inductance obviates this difficulty. To illustrate-suppose that the inductance is large in comparison to the resistance, the expression for $\beta$ will reduce to

$$
\beta=R / 2 \sqrt{C / L}
$$

It is independent of the frequency. All frequencies are attenuated alike, so that high inductance not only diminishes attenuation but also renders the circuit distortionless. Such a circuit is the ideal circuit for telephonic and telegraphic wave transmission. 
Mr. Oliver Heaviside, of England, to whose profound researches most of the existing mathematical theory of electrical wave propagation is due, was the originator and most ardent advocate of wave conductors of high inductance. His counsel did not seem to prevail as much as it deserved, certainly not in his own country. I trust that the physical view of attenuation described above in the terms of the dissipation of energy which is transmitted over the iwire will help to elucidate Mr. Heaviside's theory of high inductance wave conductors.

Wave Propagation over Non-Uniform Conductors.-But Mr. Heaviside's proposition to employ wave conductors of high inductance contained a serious difficulty which his mathematical theory was not capable of overcoming. The difficulty is this : How can a wave conductor be constructed so as to bave. a high inductance? Ordinary circuits can be endowed by as much inductance as may be required by simply introducing a coil of proper dimensions, with or without an iron core, into it. But this will never do in the case of a wave conductor; for a coil introduced that way will act by reflection as a barrier to electrical waves. Acting on a suggestion made by $\mathrm{Mr}$. O. Heariside ${ }^{1}$ in 1893, wave propagation experiments over long wave conductors containing a certain number of coils in series at periodically recurring points have actually been tried by telephone engineers; the results obtained have invariably proved most disappointing. I shall return to this point later on in cunnection with the description of experiments given further below. Suffice it to state here that all attempts to increase the inductance of a wave conductor by the introduction of inductance coils at periodically recurring points failed, because they had no mathematical theory to guide them, so as to avoid the difficulties of wave reflection by inductance coils introduced that way. It is hardly worth while to enter here into a discussion of the many other attempts at increasing the inductance of a wave conductor by devices which had neither theory nor experiment to recommend them. In fact, most of them were absurd on the face of it.

The first mathematical theory dealing with wave propagation over conductors of this kind was presented by the author before this Institute on March 22d, 1899. ${ }^{2}$ A more complete theory is given in the second part of this paper. The main features of

1 Heaviside, O._ "Electromagnetic Theory," vol. i., p. 435.

2 Pupin, M. I.- “Propagation of Long Electrical Waves;" Transactions, vol. xvi., p. 93, 1899. 
this theory are extremely simple and can be explained by a sim. ple mechanical illustration. Consider the arrangement of Fig. 1. A tuning fork has its handle $C$ rigidly fixed. To one of its prongs is attached a flexible inextensible cord $B D$. One terminal of the cord is fixed at $D$. Let the fork vibrate steadily, the vibration being maintained electromagnetically or otherwise. The motion of the cord will be a wave motion. If the frictional resistances opposing the motion of the cord are negligibly small the wave motion will be approximately that of stationary waves as in Fig. 2. The direct waves coming from the tuning fork and the reflected waves coming from the fixed point $D$ will have nearly equal amplitudes and by their interference form approximately stationary waves. If, however, the frictional
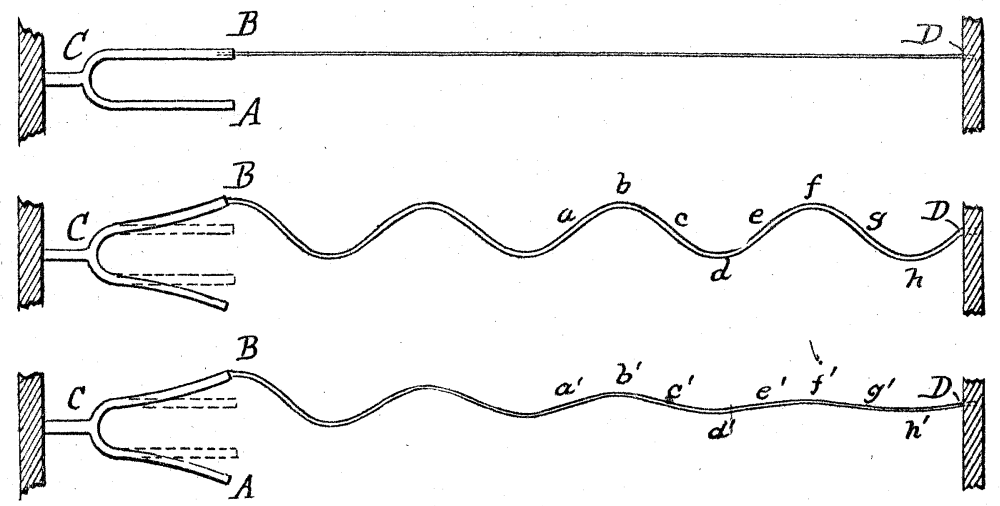

Figs. 1, 2, 3.

resistances are not negligibly small, then there will be dissipation of the propagated wave energy. Hence the direct and the reflected waves will not have equal amplitudes, and, therefore, their interference will not result in stationary waves. The attenuation of the wave is represented graphically in Fig. 3. Experiments will show that, other things being equal, increased density of the string will diminish attenuation, because a larger mass requires a smaller velocity in order to store up a given quantity of kinetic energy, and smaller velocity brings with it a smaller frictional loss. This is a striking mechanical illustration of a wave conductor of high inductance. It should be observed here that an increase of the density will shorten the wave-length.

Suppose now that we attach a weight, say a ball of beeswax, at the middle point of the string, in order to increase the vibrating mass. This weight will become a source of reflections and 
less wave energy will reach the point $\mathrm{D}$ than before. The effciency of transmission will be smaller now than before the weight was attached. Subdivide now the beeswax into three equal parts and place them at three equidistant points along the cord. The efficiency of wave transmission will be better now than it was when all the wax was concentrated at a single point. By subdividing still further the efficiency will be still more improved; but a point is soon reached when further subdivision produces an inappreciable improvement only. This point is reached when the cord thus loaded vibrates very nearly like a uniform cord of the same mass, tension, and frictional resistance. Such a loaded cord with a tuning fork attachment is represented in Fig. 4.

If an increase in efficiency of wave transmission over a cord

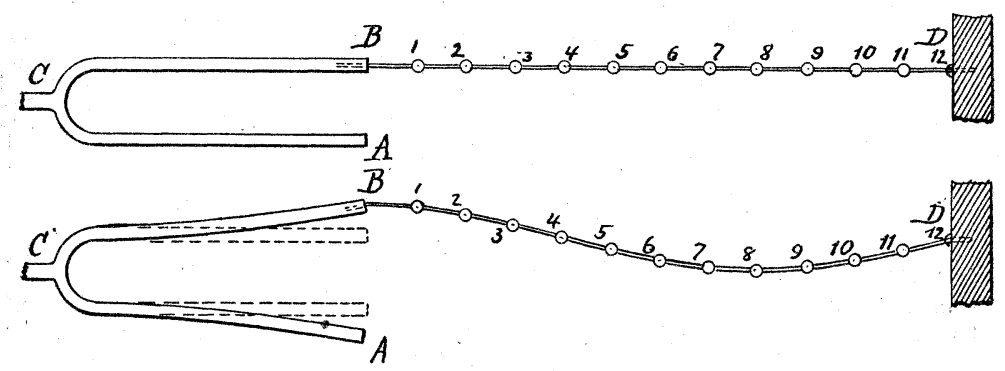

Figs. 4 and 5.

thus loaded is to be obtained, it is evident that the load must be properly subdivided and the fractional parts of the total load must be placed at proper distances apart along the cord, otherwise the detrimental effects due to reflections resulting from the discontinuities thus introduced will more than neutralize the beneficial effects derived from the increased mass.

The problem of finding the proper distance at which the loads should be placed is a definite mathematical problem of Analytical Mechanics, but unfortunately it was never solved. Fig. 5 represents a cord carrying loads at proper distances apart. Experiments with cords of this kind will soon convince one that the distance between the loads should be considerably smaller than one-half of the wave-length of the wave which is to be transmitted. So that though a given cord may be properly loaded for some wave-length it will not be properly loaded for shorter wavelengths. It is impossible to load a cord in such a way as to make 
it equivalent to a uniform cord for all wave-lengths; but if the distribution of the loads satisfies the requirements of a given wave-length it will also satisfy them for all longer wave-lengths. It should be observed now that the wave-length which is considered here is not the wave-length of the cord without the loads, but the wave-length which the frequency under consideration will have on the properly loaded cord, or what is the same thing, on a uniform cord of the same mass, tension, and frictional resistance, as the loaded cord. This point is of fundamental importance, for the wave-length corresponding to a given frequency may and generally will be much shorter on the loaded cord than on the cord without the loads.

A cord of this kind is a mechanical analogy to an electrical wave conductor. The mathematical law in accordance with which such a cord moves is the same as that in accordance with which the electrical current is distributed over the wave conductor under the action of similar forces. The reason for that is not far to seek. We have the same reactions in both cases, viz.:Kinetic or mass reaction, tensional reaction, and resistance reaction in the case of the cord. Electro-kinetic reaction, capacity reaction, and ohmic resistance reaction in the case of the wave conductor. The mathematical form of these reactions is the same in both cases, hence one is an exact analugy of the other.

The insertion of inductance coils at periodically recurring points along the wave conductor, represented in Fig. 3 of Part II., produces the same effect upon electrical wave transmission as the distribution of the small loads along the stretched cord of Fig. 4 produces upon mechanical wave transmisssion along the cord. The mathematical theory of wave propagation over non-uniform conductors of this kind is given in Sections I and II of the Second Part of this paper. This theory is, of course, at the same time the mathematical theory of wave propagation over a loaded cord described above. The main object of this theory is find an answer to the question: Under what conditions are nonuniform conductors described in these two sections equivalent to their corresponding uniform conductors? The answer which the mathematical theory gives to the question just proposed is definite. To formulate it introduce here a convenient technical term. Consider the distance between two consecutive inductance points, that is, the points at which the inductance coils are introduced. Denote it by $l$, and let the wave length which 
is to be transmitted be $\lambda$. Now introduce an angle $\varphi$ such that

$$
\varphi / 2 \pi=l / \lambda .
$$

The angle $\varphi$ shall be called the angular distance between the inductance points, or inductance sources. The angular distance $2 \pi$ corresponds to the wave-length. The law which determines the degree of equivalence between a non-uniform conductor and its corresponding uniform conductor can now be stated as follows:

A non-uniform conductor is as nearly equivalent to its corresponding uniform conductor as $\sin \varphi / 2$ is to $\varphi / 2$.

It is evident that $\varphi$ is inversely proportional to the wavelength, so that for a given distance between the reactance points the degree of equivalence diminishes as the wave-length diminishes. If a wave of complex harmonic frequency, such as occur in telephony, be transmitted over a non-uniform conductor, then the action of the conductor will be different for the different components of this complex harmonic wave. If, however, the non-uniform conductor acts with sufficient approximation as a uniform conductor toward the highest important frequency of this complex wave then its approximation to a uniform conductor will be even higher for the lower frequencies and thus for all the frequencies of the wave. A numerical example will illustrate this point more clearly. It is well, however, to state more fully the meaning of the expression "equivalence between a non-uniform conductor and its corresponding uniform conductor." All that we can predicate of a wave of a given frequency is that it has a certain wave-length and a certain attenuation constant. Hence, if a wave of a given frequency has the ame wave-length and the same attenuation constant on a nonuniform conductor as it has on the corresponding uniform conductor then the two conductors are equivalent to each other. If these two quantities differ by, say, three per cent., then an approximate equivalence up to within three per cent. exists.

Consider now the following numerical example: A twin conductor, such as employed for telephone cables, has a length of 250 miles. Let its constants have the following values per mile:

Inductance $=0$.

Resistance $=9$ ohms.

Mutual capacity $=.074$ Microfarads.

According to the high but definite standard which the New 
York Telephone Company employs the limiting-distance of telephony over such a cable is 39 miles. According to the lower standard which is maintained in long-distance telephone work the limit would be 78 miles. The results obtained by me experimentally seem to verify these figures. I find that at a distance of one hundred miles telephony over such a cable is very poor, in fact, impracticable, and at a distance of 125 miles impossible. It is proposed now to decrease attenuation and distortion over such a cable by the insertion of inductance coils at periodically recurring points. The attenuation constant $\beta$ in this case is given by the formula ${ }^{1}$

$$
\beta=\frac{R}{\sqrt{2}} \sqrt{\frac{C}{L}}
$$

Say that it is required to have $\beta=.015$. Assume that the introduction of the inductance coils adds 9 ohms per mile, so that $R=$ 8 ohms. These values of $R$ and $C$ require $L=.056$ henrys. The attenuation factor at a distance of 250 miles would be $e^{-250 \beta}=\frac{1}{40}$, (roughly). That is, $2 \frac{1}{2} \%$ of the current leaving the transmitting end will reach the receiving end. This is quite sufficient for telephonic purposes; but it should be observed that better efticiency of transmission could be obtained by making $L$ larger. Notice now that the attenuation factor for this cable without the coils would be, for a frequency of 600 p.p.s., roughly, $\frac{1}{25 \times 10^{4}}$ that is, with a given initial current, the current at the receiving end would be 6000 times larger with the coils than without them.

The next step is to find the wave-length for the highest important frequency in telephony over a uniform wave conductor. having $L=.056$ henrys, $R=18 \mathrm{ohms}, C=.074$ microfarads. The best telephone practice assumes that 750 P. P. s. is the highest frequency of any importance. The wave-length corresponding to this frequency over a uniform conductor of this description is obtained from the formula ${ }^{1}$

$$
\lambda=\frac{2 \pi}{p \sqrt{2 L C^{\gamma}}}=14.6 \text { miles }
$$

approximateiy.

1 See Equation (4a), Sect. I., Part II. 
Suppose now that at each mile we place a coil of inductance $L=.056$ henry and a resistance $R=9$ ohms. The angular distance $\varphi$ for frequency 750 P. P. s. of the non-uniform conductor thus obtained will be $2 \pi / 14.6$. The degree of equivalence of this non-uniform conductor to its corresponding uniform conductor is measured by the degree of equivalence of $\sin \pi / 14.6$ and $\pi / 14.6$. Now $\sin \pi / 14.6$ differs from $\pi / 14.6$ by less than one per cent. of the value of $\pi / 14.6$; hence for a frequency of 750 P. P. s. the wave-lengih and the attenuation constant on the non-uniform conductor will differ from the wave-length and attenuation constant on the corresponding uniform conductor by less than one per cent. of the values of these constants. Such a difference cannot be detected by any of the experimental methods which are at present available for investigating wave propagation. In telephonic transmission the ear could not detect it. For lower frequencies the differences will be even considerably smaller. Hence, the non-uniform conductor thus obtained will represent a uniform, non-attenuating, distortionless conductor for telephonic transmission.

It should be observed here that in the case of a submarine cable of say 2,000 miles the attenuation constant should be much smaller than the value of $\beta$ given above, in order to have a sufficiently sinall attenuation factor. Now the capacity per mile of a submarine cable is about four times as large as the capacity of the telephone cable just described. Hence, both on account of the long distance and also on account of the much increased capacity, the inductance per mile will have to be much larger than in the case just discussed. But high inductance and large capacity will give a very short wave-length. For instance, if in the case of the submarine cable having six times the capacity we employ an inductance six times as large as in the case of the telephone cable we shall obtain for the frequency of 750 P.P.s. a wave-length of only $14.6 \div 6=$ 2.43 miles. Hence, since the inductance coils will have to be placed apart at one-sixth of the distance employed in the case of the telephone cable, the distance between them will be about 880 . feet. The distance between the inductance coils depends entirely on the circumstances of each particular case. But in all cases the rule given above contains the necessary and sufficient directions.

This rule and the method based upon it are recommended by the mathematical theory developed in Part II of this paper for the construction of distortionless wave conductors of high efficiency 
of transmission. It will be shown now in how far this theory is confirmed by experiment.

\section{Experimental Part.}

a. Description of the Experimental Cable.-A so-called artificial cable possessing the above constants was constructed by me for the purpose of testing experimentally the mathematical theory developed in Section III of Part II. Previous experiments in this line will be found in the author's paper of March 22d, 1899, cited above.

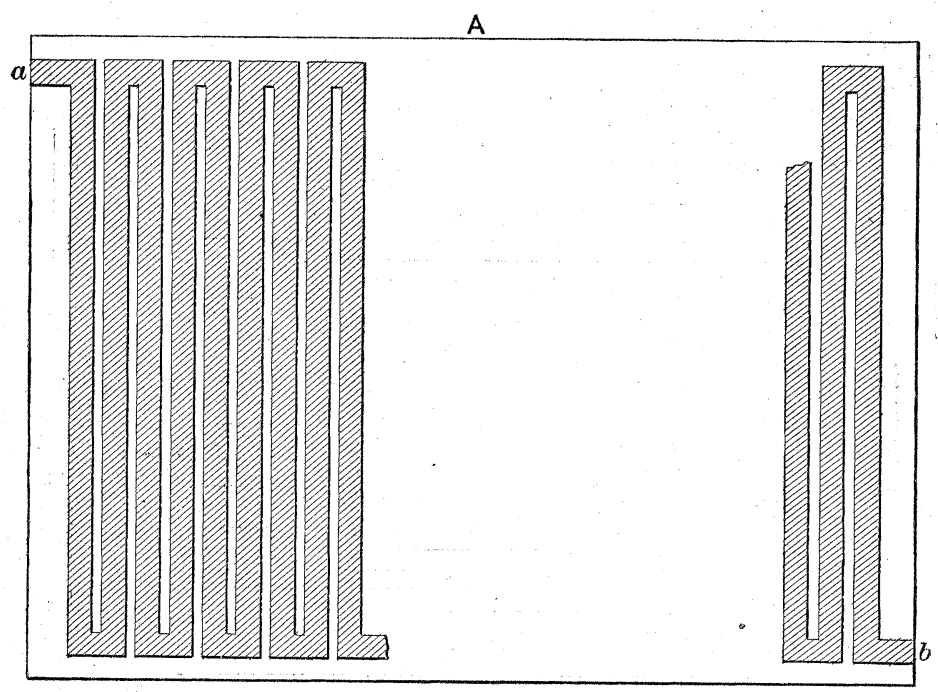

FIG. 6.

The cable has 250 sections. One of these sections is represented in Fig. 6. It consists of a sheet $A$ of paraffined paper. On each side of this sheet is a strip of tinfoil $a b$. The resistance of this strip is approximately 9 ohms. The capacity of the condenser formed by the two strips is .074 microfarads approximately. 250 sections of this kind connected in series represent a cable of 250 miles in length having a resistance of $9 \mathrm{ohms}$ and a capacity of .074 microfarads per mile. That such is actually the case was verified experimentally, as will be shown presently. In Fig. 8 the straight lines $\mathrm{A} B \mathrm{~B} \quad \mathrm{D} \quad \mathrm{A}_{1} \mathrm{~B}_{1} \mathrm{C}_{1} \mathrm{D}_{1}$ represnt these sections. The upper lines represent one side of the cable, and the lower lines represent the other side. The sections can be con- 
nected in series by couplers to which these sections are attached. One of these couplers is represented in Fig. 9. A wooden strip $A B$ has holes drilled through it at regular intervals. Through each hole passes a bolt $c$, the diameter of which is smaller than the diameter of the hole. Two hard rubber washers $a$ and $b$ hold the bolt in place and prevent it from touching the wood. A nut holds the brass plates $d$ in place. Two consecutive brass plates $d d$ can be conductively connected by the plug $e$. Screws, as indi-

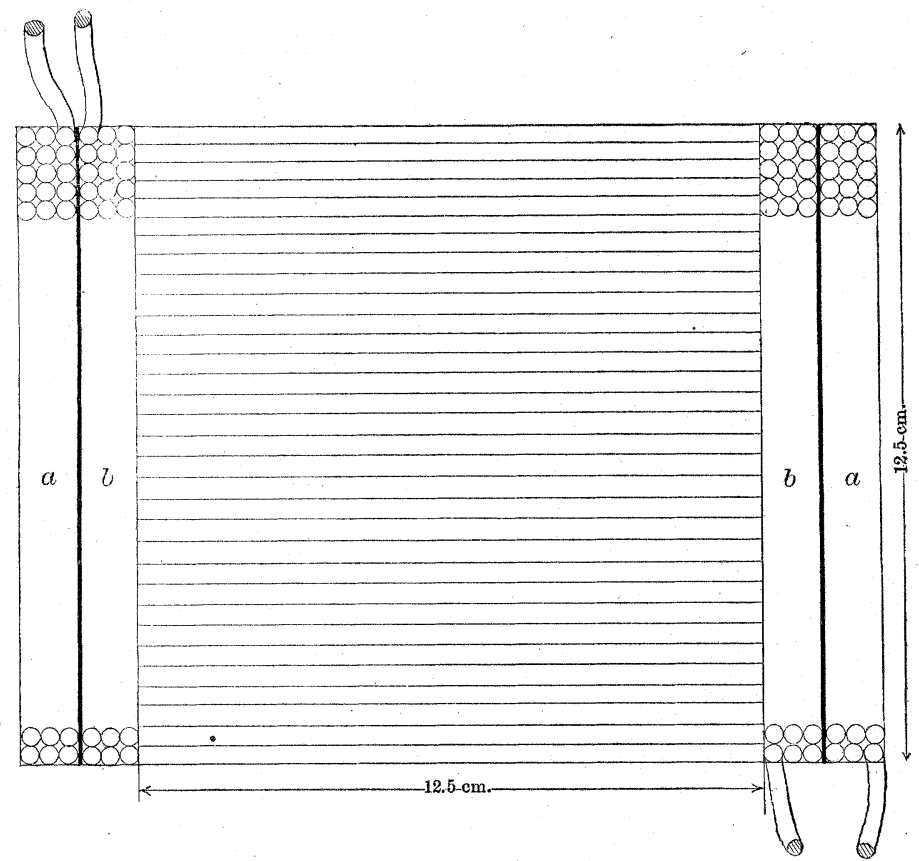

Fig. $\%$.

cated, connect the terminals $f f$ of the condenser sections and of the coils $g g$ to the brass plates. These brass plates are indicated in Fig. 8 by numerals $1,2,3, \ldots$ When the plugs are out, then the consecutive sections of the cable are connected to each other by the inserted coils (see Fig. 8). The plugs being in the coils are short-circuited, and the consecutive sections of the cable are connected to each other directly by the plugs. In this case we have a uniform cable; in the former case we have a non-uniform cable of 250 miles length, with inductance coils inserted at each mile. The coils are wound as represented in Fig. 7. The twin coils $a a_{1}, b b_{1}, c c_{1}$ of Fig. 8 are represented in Fig. 7 by coils $a a$ and $b b$. 
These two coils are wound on one spool, and are separated by a sheet of cardboard indicated by a black line in Fig. 7 which is 1-64th of an inch thick. The coil when finished is boiled in beeswax at a temperature of about $280^{\circ}$ Fahrenheit, in order to drive all the moisture out and insure good insulation. The dimensions of the coil are given in Fig. 7. Each coil had 580 turns of No. 20 wire B. \& S. The average inductance of one of these coils is .030 henry, and the mutual inductance .028 henry. Each coil, therefore, when connected into the line, as indicated in Fig. 8, has an effective inductance of

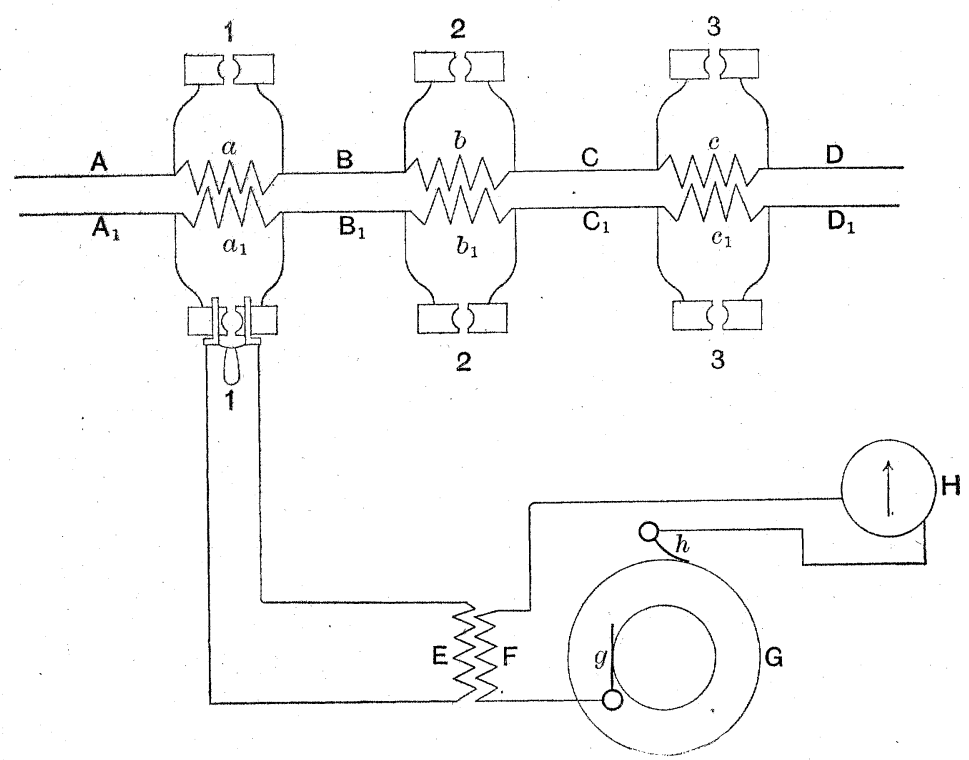

Fig. 8.

.058 henry. This method of bringing the corresponding coils of the two sides of the cable into close relation by mutual induction is not necessary; it is a matter of convenience and economy.

The cable sections are divided in five groups of 50 each, and each of these groups is enclosed in a separate box. Each box represents, therefore, 50 miles of the cable. Fig. $10 \mathrm{a}$ is a reproduction from the photograph of one of these boxes. The box marked $\mathrm{A}$ is the cable box. On two opposite sides are five rows B... of binding posts, each row having 10 pairs. They 
are the terminals of the condenser sections. From these binding posts double cotton covered wires which have been boiled in beeswax lead to the row of brass plate couplers (Fig. 9) indicated in the picture by letters $a, b, c, \ldots$ These boxes were made for me by the well-known mechanician, Mr. E. V. Baillard, to whom my sincere thanks are due for the keen interest which he took in this exceedingly laborious work. The rest of the work on the cable was done in the Columbia University laboratory for electromechanics. The insulation per section was about 50 megohms.

b.-Theory of the Experimental Method.-The mathematical theory of Section III, Part II, states that up to a frequency of 750 p.p.s. the waves will have to within an approximation of one

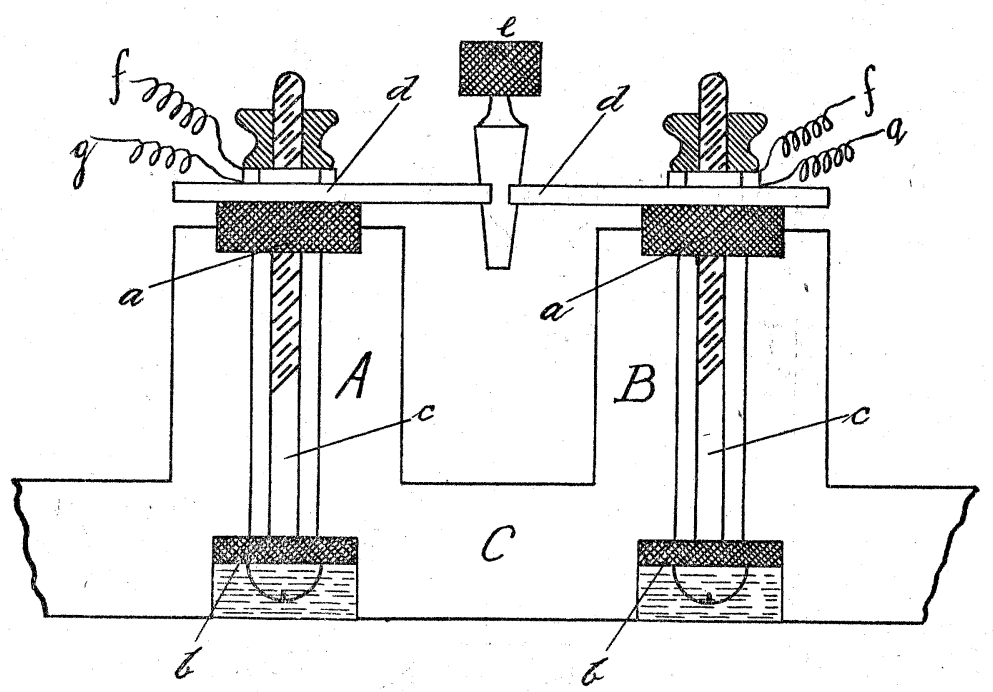

Fia. 9.

per cent. the same wave-length and the same attenuation constant on the cable just described as on its corresponding uniform conductor. This is the side from which experiment has to approach the theory. The method employed in this investigation has, therefore, for its object the determination of wave-length and attenuation constant. Its theory can be stated as follows:

The current $\eta$ at any point $\xi$ of a uniform loop can be expressed. ${ }^{1}$

1. M. I. Pupin:-Propagation of Long. Electrical Waves, equ. (7); TransTACrons, vol. xvi., p. 93, 1899. 
1900.]

$$
\eta=A\left\{e^{\beta \xi} \sin (p t-\alpha \xi)+e^{-\beta \xi} \sin (p t+\alpha \xi)\right\}
$$

Let $M(\eta)$ denote the mean value of the current between $t={ }^{-} t_{1}$ and $t=t_{1}+T / 2$, then

$$
M(\eta)=\frac{y_{0}}{2}\left(e^{\beta \xi}+e^{-\beta \xi}\right) \cos \left(p t_{1}-\alpha \xi\right)
$$

Here $\xi$ is the distance from the middle point of the loop. The time is counted from the moment when $\eta$ is zero at the

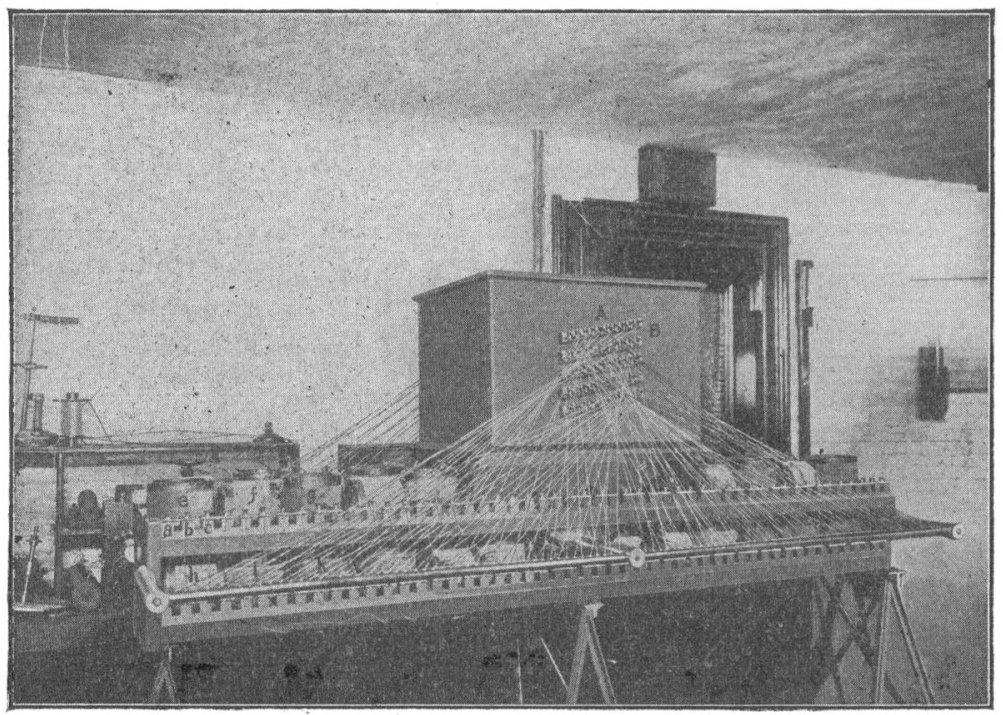

Fig. $10 \mathrm{~A}$.

middle point. If we then determine the mean values from the moment when $\eta$ vanishes at the middle point we shall have $t_{1}=$ 0 and

$$
M(\eta)=\frac{y_{0}}{2}\left(e^{\beta \xi}+e^{-\beta \xi}\right) \cos \alpha \xi
$$

or since $\lambda=2 \pi / \alpha$, where $\lambda$ is the wave-length

$$
M(\eta)=\frac{y_{0}}{2}\left(e^{\beta \xi}+e^{-\beta \xi}\right) \cos \frac{2 \pi}{\lambda} \xi
$$


$y_{0}$ is evidently the mean value of the current at the middle point. This formula forms the basis of the experimental method employed here. From it the wave-length and the attenuation constant were determined.

c. Determination of the Wave-length.-1. To investigate the wave-length and the attenuation constant of a given frequency it was necessary to impress a simple harmonic electromotive force upon the cable. This was obtained as follows:- A small alternator of thirty poles running at a normal speed of 2400 revolutions per minute was employed. It gives a complex harmonic e. M. F. in which the third and fifth harmonic are quite strong. At the normal speed the frequency of the fundamental was 600 p.p.s. By transformation as indicated in Fig. 10 the upper

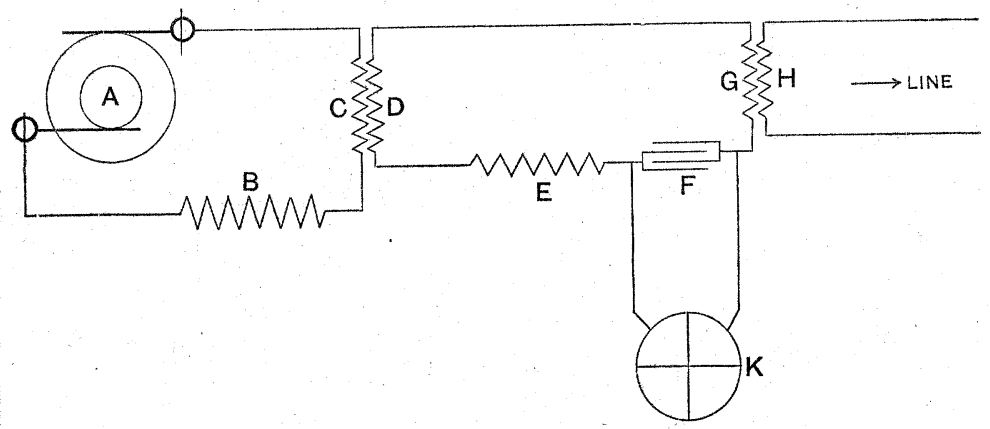

Fig. 10.

harmonics were weeded out by tuning, In this diagram $\mathrm{A}$ is the alternator. The secondary circuit D, E, F, G, contains a condenser $\mathrm{F}$, and an auxiliary coil E. By adjusting the capacity of condenser $\mathrm{F}$ and the inductance of coil $\mathrm{E}$, the impedance of this secondary circuit for the fundamental frequency can be reduced to a minimum, in which case the fundamental current in this circuit is by far predominant. Dnder these conditions the E. M.F. impressed upon the tertiary coil $\mathrm{H}$, which connects to the line, is very nearly simple harmonic. This was verified by plotting experimentally the E. M. F. curve in the tertiary circuit. The point of resonance was detected by connecting the terminals of condenser $\mathrm{F}$ to a multicellular voltmeter $\mathrm{K}$ and watching for the maximum rise of the potential. While the observations which will be described presently were made the reading of this voltmeter was continually watched. A change in it indicated a change in speed 
and impressed E. M. F. and the observations had to be suspended until the voltmeter returned to the same reading. Considering the variability of the electrical pressure in a University plant where every one of the many departments draws its current from the same source, it will be readily seen that these observations were somewhat trying.

2. To determine the mean value of the current a Townsend contact-maker ( $G$, Fig. 8) was ased. ${ }^{1}$ Its circumference was divided into thirty equal parts, since the alternator had thirty poles. Every other section of the circumference was connected to a ring $g$. This ring and the circumference were connected to the rest of the circuit, $g F H h$, by two brushes $g h$. The contact-maker was mounted on the shaft of the alternator. The time interval, of half a period, during which the contact lasted, could be shifted by shifting the brush $h$ along the circumference of the contactdisk. $\quad I$ is a Rowland-D'A rsonval galvanometer suitably sr unted so as to keep the readings within a desirable range. The current readings were obtained as follows: A two-pole contact springjack (1, Fig. 8) was inserted across the terminals of an inductance coil, say $a_{1}$, as indicated. The coil was thus shunted by circuit $1 E$. This circuit contained a small coil $e$ and one or two ordinary 16-c.-p. incandescent lamps in series with it. The coil acted inductively upon the circuit containing the contact-maker and the galvanometer. A simple consideration will show that the galvanometer reading was proportional to the mean value of the current flowing through the inductance coil $a_{1}$ during the halfperiod corresponding to the position of the brush $h$. Suppose now that this brush is set in such a way as to give the maximum reading when the contact-spring-jack is across the inductance coil at the middle point of the cable. Then, as shown in equation(1), the reading at any other inductance coil will be proportional to

$$
\left(e^{\beta \xi}+e^{-\beta \xi}\right) \cos \frac{2 \pi}{\lambda^{\circ}} \xi
$$

where $\xi$ denotes the distance of the inductance coil under consideration from the middle point. This expression passes through zero whenever $\xi$ is an odd multiple of the quarter wave-length, and the reading passes then from one side of the zero position to the other. The brush $h$ was, therefore, set so as to give the maxi-

1 See Transactions, vol. xvii., No. 1, Jan., 1900. 
mum reading at the middle point of the line. Then the springjack was run along the line and the sign of the readings noted at $1,2,3, \ldots$ etc. The number of reversals of readings corresponding to a given length of the line was counted.

The reversals occur whenever $\xi$ is an odd multiple of $\lambda / 4$. Suppose that five reversals occurred in the distance of 41 miles. It ineans that $9 / 4$ wave-lengths cover the distance of 41 miles; hence the wave-length is

$$
\lambda=18.2 \text { miles. }
$$

This was found to be the case with a frequency of 600 P. P. s. The wave-length calculated from the formula

$$
\begin{aligned}
& \lambda=\frac{2 \pi}{p \sqrt{2 L C}} \\
& p=2 \pi \times 600 . \\
& L=.058 . \\
& C=.074 \times 10^{-6}
\end{aligned}
$$

gives $\quad \lambda=18.1$ miles.

The agreement between the calculated and the olserved wavelength was extremely good for this frequency, considering the inequality of the cable sections which necessitates taking for $L$ and $C$ average values. Following is a table of wave-lengths for other frequencies, giving both the observed and the calculated values.

TABLE OF WAVE LENGTHS.

$\begin{array}{cll}\text { FREQUENCY. } & \lambda_{\text {obs. }} & \lambda_{\text {calc. }} \\ 625 & 17 & 17.3 \\ 600 & 18.2 & 18.1 \\ 450 & 24 & 24.1 \\ 260 & 41.4 & 41.7 \\ 245 & 44 & 44.4 \\ 230 & 48 & 48.2\end{array}$

At the frequency of 600 P.P.s. the non-uniform cable has 18 coils per wave-length. At the lower frequencies the number of coils per wave-length is larger, and therefore the cable approximates then its corresponding uniform conductor even more closely 
than at the frequency of 600 P.P.s. But the approximation at this higher frequency is already so close that no difference in the behavior of the cable at the various frequencies experimented with and given above could be detected experimentally. As far as the wave-length determination is concerned the agreement between theory and experiment is very satisfactory.

3. To determine the wave-length on the cable without the coils plugs (see $e$, Fig. 9 ) were put in $1,2,3 \ldots \ldots$ The readings were taken as follows: The two-pole spring-jack was inserted across a

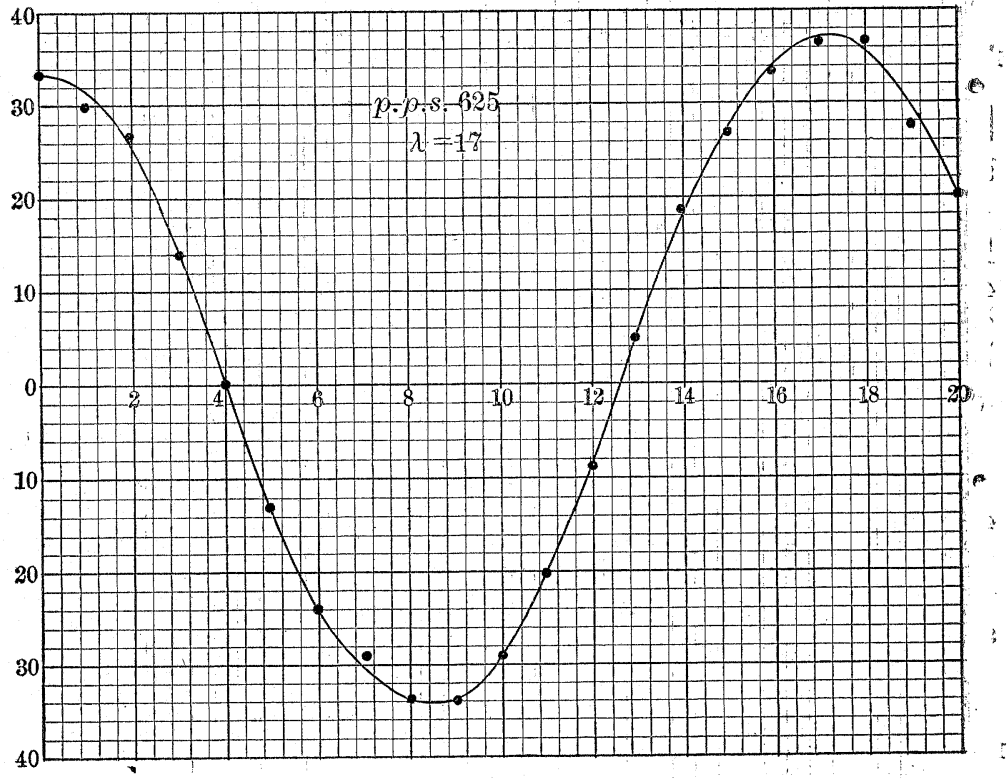

FIg. 11.

pair of plates, say 1. Then the short-circuiting plug (see e, Fig. 9) was taken out and the deflection in the galvanometer noted. The number of reversals was counted as before, and from these the wave-length was estimated. The value of the wave-length thus determined for a frequency of 600 P.P.s. was 126 . Its calculated value was 125. Thus the insertion of the coils reduces the speed of propagation to one-seventh of its original value since it shortens the wave-length to that extent.
d. Determination
of : the
Attenuation
Constant - First 
Method.-It was shown above that the equation of the curve of the mean value of current is

$$
y=\frac{y_{0}}{2}\left(e^{\beta \xi}+e^{-\beta \xi}\right) \cos \frac{2 \pi}{\lambda} \xi
$$

where $y$ is the mean value of the current at the distance $\xi$ from the middle point of the cable and $y_{0}$ is the mean value at the

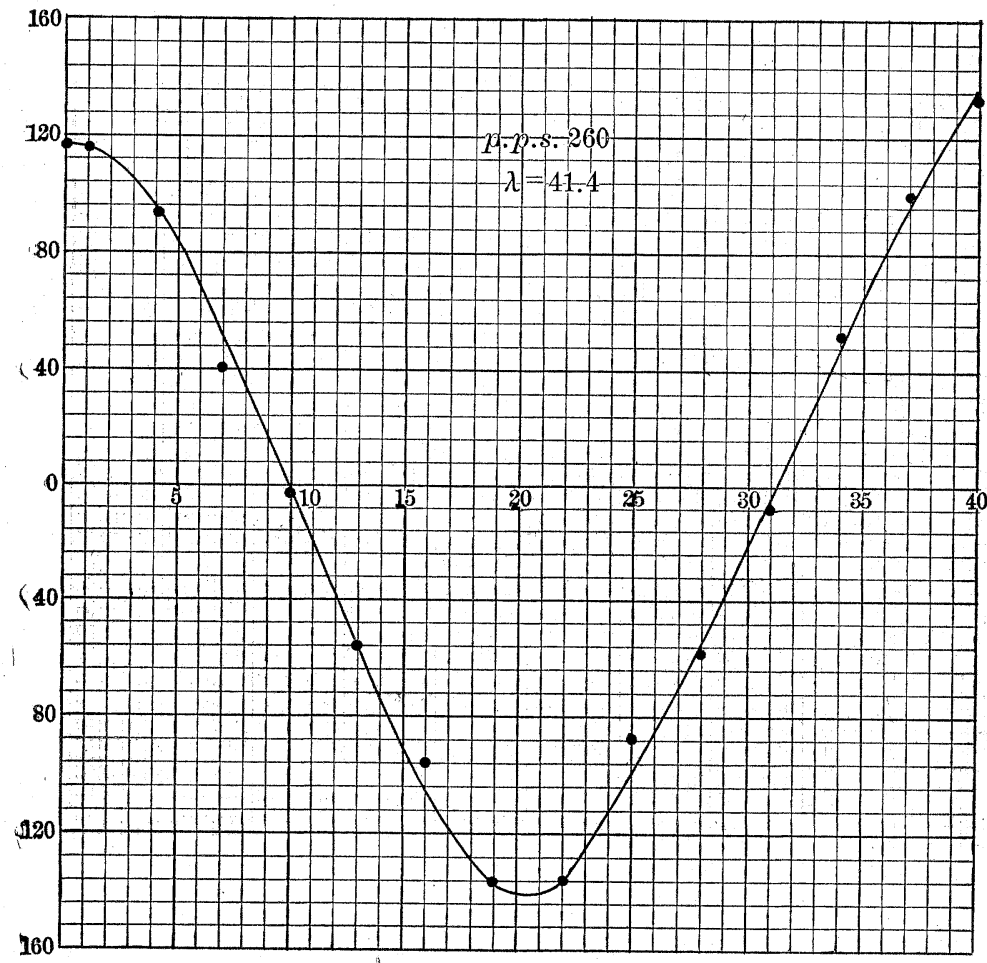

Fig. 12.

middle point when the brush $h$ of the contact-maker is set to give a maximum deflection at this point. In Fig. 11 and Fig. 12 two such curve are given. In these curves the abscissæ represent the number of the coil at which the reading was taken, and the ordinate represents the galvanometer reading at the corresponding coil.

An attempt was first made to determine $\beta$ from these curves. 
Let $y_{\frac{\lambda}{2}}$ be the value of $y$ at $\xi=\frac{\lambda}{2}$, then

$$
y_{\frac{\lambda}{2}}=-\frac{y_{0}}{2}\left(e^{-\frac{\beta \Lambda}{2}}+e^{-\frac{\beta \Lambda}{2}}\right)
$$

Since $\frac{\beta \lambda}{2}$ is small we can also write

$$
y_{\frac{\lambda}{2}}=-\frac{y_{0}}{2}\left(2+\frac{1}{4} \beta^{2} \lambda^{2}\right)
$$

Therefore considering the numerical values, only, of $y_{0}$ and $y_{\lambda}$ we shall have

$$
\beta=\frac{2}{\lambda} \sqrt{\frac{2\left(y_{\frac{\pi}{2}}-y_{0}\right)}{y_{0}}}
$$

Referring now to curve Fig. 11

$$
\begin{aligned}
\lambda & =17 \\
y_{0} & =33.5 \\
y_{\frac{K}{2}} & =34
\end{aligned}
$$

$$
\frac{2}{\lambda} \sqrt{\frac{2\left(y_{\frac{\kappa}{2}}-y_{0}\right)}{y_{0}}}=\frac{2}{17} \sqrt{\frac{1}{33.5}}=.02=\beta
$$

The value of $\beta$ calculated from the formula

$$
\begin{gathered}
\beta=\frac{R}{\sqrt{\overline{2} 10^{3}}} \sqrt{\frac{\bar{C}}{L}} \\
\text { gives } \beta=.0141
\end{gathered}
$$

if we assume for $L, C$, and $R$ the following average values: $L=.058, C=.074 \times 10^{-6}, R=17.5$.

The question now arises: What degree of accuracy can be claimed for this experimental determination of $\beta$ ? The expression for $\beta$ contains the difference of quantities which are 
nearly equal. Now this difference is very near the limit of the errors of observation, the limit being quite considerable on account of several reasons. The chief reason is this. The condenser sections of the cable are far from uniform. This lack of uniformity is due, first to the varying thickness of the tinfoil which makes the resistance vary from section to section. Secondly, the sections are heated before compression is applied to them. This heating is of course not quite uniform; the amount of paraffin driven out by the applied pressure is, therefore, different in different sections. Hence the variation of capacity. The result is that the speed of propagation is different in different sections. The difference may amount to as much as 20 per cent. Hence the wave-length will change considerably from point to point of the cable. This will be observed in the curves of Fig. 11 and Fig. 12. Thus in Fig. 11 the first quarter wave-length is four miles long, the second quarter wavelength is 4.5 miles and the average is 4.25 . The same variation will be observed in curve of Fig. 12. It is evident, therefore, that the method described above for taking readings at the various points of the cable will not give readings which will follow very strictly the mathematical law of the formula on page 22. Taking all these things into consideration, it is evident that this method just pointed out for determining experimentally the attenuation constant, is not strictly applicable to our cable.

Another method will be described now which determines the attenuation constant not frorn the one-half wave-lengths, only, near the middle of the line, but takes all the waves corresponding to a long length of the line into consideration. The wavelength determination given above agreed so much better with the theory for the very reason that the method employed gave the average wave-length and not the wave-length at any particular point of the cable.

Although the curves of Figs. 11, 12 and 13 do not enable us to calculate $\beta$ accurately they are still of considerable importance. First, they show that the cable, though it has a large resistance, possesses, nevertheless, a small attenuation constant. This is particularly well shown in the curve of Fig. 13. This carve represents the mean square of current curve at the the various coils. The readings were obtained in this case by dispensing with the sliding contact arrangement and putting in place of $E$ an electrodynamometer. This mean square curve represents a very near 
approach to the stationary wave form, which shows that the attenuation is extremely small. Secondly, the smoothness of the curves 11,12 , and 13 shows that the non-uniform cable acts, even at the frequency of 625 p. .. s., just like a uniform cable. This, in fact, was the principal aim of this experimental research.

Second Method of Determining $\beta$.-The value of $\beta$ was determined from

$$
y=\frac{y_{0}}{2}\left(e^{\beta \xi}+e^{-\beta \xi}\right) \cos \frac{2 \pi}{\lambda} \xi
$$

as follows: Suppose that at a considerable distance from the

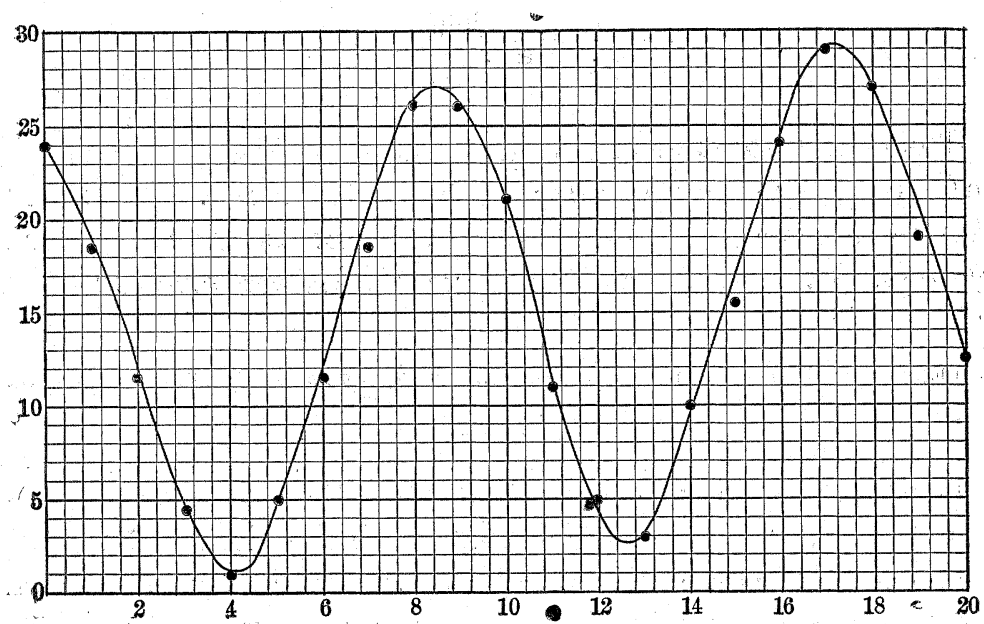

FIG. 13.

point $\xi=0$ we find a point the distance of which is some mul tiple of $\lambda / 2$. Then

$$
y_{1}=\frac{y_{0}}{2}\left(e^{\beta \xi_{1}}+e^{-\beta \xi_{1}}\right)
$$

This was done experimentally by shifting the brush $h$ (Fig. 8) until the maximum reading was obtained at the middle point of the line. It was 152 scale divisions-that is, $y_{0}=152$. Then the spring-jack was carried to the beginning of the cable and passed alung from coil to coil until the galvanometer reading passed through a maximum. The distance $\xi_{1}$ of this point from $\xi=0$ was 145 . The reading actually obtained was 60 , but this reading 
was obtained from a shunt wire, and the reading as calculated from the shunt was 630 . Now, when $\xi_{1}$ is so large that the omission of $e^{-\beta \xi_{1}}$ in comparison to $e^{+\beta \xi_{1}}$ involves an error of the same order of magntude as the errors of observation, we have the simplified formula

$$
y_{1}=\frac{y_{0}}{2} e^{\beta \xi_{1}}
$$

Therefore,

$$
\beta=\frac{1}{\xi_{1}} \log \frac{2 y_{1}}{y_{0}}
$$

In the case before us

$$
\beta=\frac{1}{145} \log \frac{1260}{152}=.0145
$$

The calculated value of $\beta$ as given above is .0141. Experiment and theory agree quite satisfactorily. The equivalence between the non-uniform cable and its corresponding uniform conductor up to 625 p.p.s. has therefore been established experimentally.

Internal Reflection of Electrical Waves in a Non-dniform Cable.

4

It remains to be shown now that when the distribution of the inductance coils over the cable does not render the cable approximately equivalent to its corresponding uniform conductor, then there will be serious internal reflections which will diminish the efficiency of wave transmission.

For this purpose the inductance coils were connected in groups of ten; one of these groups was inserted into the cable every ten miles, so that the total inductance of the cable was now the same: as before. A simple harmonic E. M. F. of 260 p. p.s. was impressed upon the cable. The wave-length of a wave of this frequency on the corresponding uniform conductor was found (see Fig. 12) to be 41.4 miles so that the inserted inductance coils were apart at a distance which is equal to about one-quarter of the wave-length on the corresponding uniform conductor. The degree of equivalence between this non-uniform cable and its corresponding uniform conductor is the same as the degree of equivalence between $\sin \pi / 4$ and $\pi / 4$; that is to say, the resemblance between the two is very small. 
Theory of the Experiments on Internal Wave Reflections. -The current at any point between two consecutive groups of coils can be written as follows:

$$
y=A e^{\beta \xi} \sin (p t-\alpha \xi)+B e^{-\beta \xi} \sin (p t+\alpha \xi)
$$

that is, there are two waves, one a direct one, and the other reflected one. This equation can also be written:

$$
y=\sqrt{A^{2} e^{2 \beta \xi}+B^{2} e^{-2 \beta \xi}+2 A B \cos 2 \alpha \xi} \sin (p t-\varepsilon)
$$

where

$$
\tan \varepsilon=\frac{A e^{\beta \xi}-B e^{-\beta \xi}}{A e^{\beta \xi}+B e^{-\beta \xi}} \tan \alpha \xi
$$

Let $M(y)$ stand for the mean value of the current, then

$$
M(y)=\frac{1}{\pi} \sqrt{A^{2} e^{2 \beta \xi}+B^{2} e^{-2 \beta \xi}+2 A B \cos 2 \alpha \xi} \cos \left(p t_{1}-\varepsilon\right)
$$

This is a maximum when $t_{1}$ is given such a value that $p t_{1}-\varepsilon$ $=o$. In that case $M(y)$ is proportional to the square root of the mean square of the current.

Experiment.-The curve of $M(y)$ was obtained in the same manner as the curves of Figures 11 and 12, except in this case the movable brush $h$, Fig 8 , was shifted until the galvanometer showedl the maximum deflection at every reading. These readings were plotted as ordinates of curve Fig. 14. Points $a b c d$ on the axis of abscissæ mark the positions of the groups of inductance coils. The effect of reflections is shown in a very marked manner by the sharp corners and strong depressions. The sharp corners coincide, of course, with the positions of the coils. The rapid rise of the minima points show strong attenuation. To compare the attenuation in this case with the attenuation which exists where the coils are distributed one per mile it is only necessary to obtain from curve 14 the curve of mean square of current and compare it to curve of Fig. 13. Draw then a straight line through two consecutive minima and compare the slope of this line in curve 13 to that of curve 14 . It will be found that the 
slope in this latter case is very much more rapid, and, therefore, the attenuation is very much more powerful; and it should be remembered that in curve of Fig. 14 we have the attenuation which is accompanying a frequency of 260 p.p.s. With a frequency of 625 p.p.s. (the frequency of Fig. 13) the attenuation would be even much more powerful. I intend to continue this particular spart of the research in the very near future.

Telephony Over the Non-Uniform Cable.-These experimental results leave no doubt as to the correctness of the prediction which the mathematical theory of Part II makes with regard to telephony over non-uniform cables. Actual experimental tests with transmitters and receivers which were kindly put at my disposal by the New York Telephone Company, for which I feel very grateful to them, verified these predictions completely. Telephonic communication over the whole of the cable was carried on with perfect ease. Both the volume and the articulation of the transmitted sound were all that could be desired. If, however, the inductance coils were cut out and the cable used as a uniform cable, then the transmission was good up to 50 miles, fair up to 75 miles, impracticable at 100 miles, impossible at distances over 112 miles. Increased distance interferes with the transmission over the uniform cable, not only on account of the diminished volume of the sound transmitted, but also on account of the rapid loss of articulation. This manifests itself at first as an apparent lowering of the pitch of the voice just as the theory demands it. My assistant's voice is of an ordinary pitch, if anything a little above the pitch of the average man's voice. When it was transmitted over 75 miles of the uniform cable (that is, the coils being disconnected from it) it sounded like a strong baritone. Over a distance of 100 miles it became so drummy, (this is an expression borrowed from telephone engineers) that it was difficult to understand it, unless nothing but ordinary conversation was carried on and then very slowly, and the speaker had to put his mouth as close to the transmitter as possible and speak out as if he were addressing a big audience. At distances over 112 miles nothing but the lowest notes of the voice could be heard; the articulation was entirely gone. When the coils were all -in, then no drumminess in the voice was noticed, and the conversation could be carried on as rapidly as one chose to do. I understand that in long distance work three galvanic cells of the same type of which I had two only were formerly employed by the 
American Telegraph and Telephone Company when they employed the old system of transmission.

Effect of internal reflections upon telephonic transmission.-The effect of internal reflection is illustrated even more strikingly by telephonic transmission than it is by the exploration of the simple harmonic wave as represented in Fig. 14. The same distribution of coils was used for telephonic transmission as described above in connection with the internal reflection experiments. A distance of a hundred miles of the cable was employed.

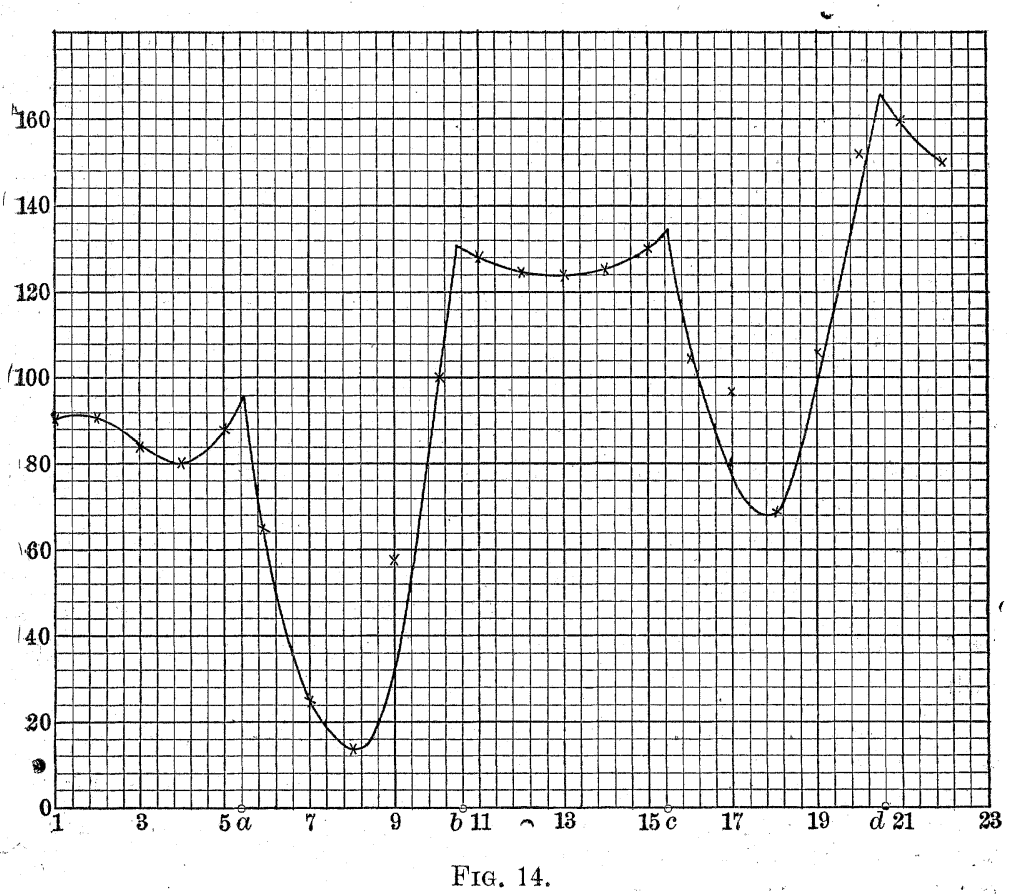

Telephonic transmission over this distance was absolutely impossible, although the lowest note in the voice was transmitted quite vigorously, and sounded like the muffled beating of a big drum. Another distribution of the coils was now tried. The coils were divided into groups of five, and these groups were put into the cable at points five miles apart. Transmission over a distance of a hundred miles became intelligible, though with much difficulty; but it was not as good as over the cable without the coils. The drumminess was even more excessive in the for- 
mer case than in the latter. One had to put his mouth very close to the transmitter, speak very slowly, very loud, and about very ordinary things, otherwise the meaning of the transmitted intelligence would not be caught at all. But if the same distance of cable was employed with the inductance coils placed one mile apart as the mathematical theory suggests then the voice of the sender sounded just as if he stood right before the receiving operator. It was not necessary to stand near the transmitter at all, in order to be heard at the other end. There was no difficulty in making the receiving operator understand even if the transmitting operator stood anywhere in the room at a distance of twenty feet or more from the transmitting instrument. The effect of the inductance coils properly distributed over the cable upon the efficiency of transmission astonishes even one like myself who have now worked for years upon and made myself perfectly familiar with this fascinating problem of ElectroMechanics.

The inductance coils which I have so far described contain no iron. Coils with iron cores to be used for this purpose will have, of course, to be constructed in a perfectly definite way to work satisfactorily. I expect to bring this matter before the INsTiTuTE at some future time. Suffice it to state now that such coils would be required in the construction of non-uniform submarine and underground telephone cables on account of the much smaller volume per unit of inductance. For long distance air lines induc tance coils without iron cores would be in many respects preferable.

In conclusion I wish to thank my assistant Mr. Cushman, an my students, Mr. Frank and Mr. Koscherak for the very faithful and persevering way in which they have assisted me throughout these experimental researches.

Laboratory for Electro-Mechanics,

Columbin, University,

New York. 


\section{PART $11 .{ }^{1}$}

Wave Propagation over Non-dniform Electrical Conduotors.

\section{INTRODUCTION.}

The main object of the inathematical theory developed in this paper is the analysis of the propagation of electrical waves over a conductor represented in Fig. 3, Sec. III. This conductor consists of a loop of wire, $L_{1} \ldots L_{\mathrm{k}+1}$ of length $2 l$, in which there are at equi distant points, the so-caller reactance points, a certain number of equal coils interposed dividing the loop into a number of equal parts, called the interstices of the loop. These coils may have condensers in series with them, or they may have secondary circuits with condensers. The propagation of electrical waves over a periodically loaded loop of this kind is compared to that over a uniform loop having the same total inductance, resistance, and capacity. This uniform loop is called "the corresponding uniform conductor" of the periodically loaded loop.

A similar problem in Mechanics is that of forced and of free vibrations of a periodically loaded heavy, flexible, inextensible string of finite length taking frictional resistance into account. To my knowledge, neither the electrical problem nor its corresponding mechanical problem have been investigated before.

It will be observed in the course of this paper that a study of the propagation of electrical waves over a periodically loaded conductor of this kind suggests forcibly an electromagnetic theory of emission and absorption of light by molecular complexes, which, on account of the physical conception underlying it. if for no other reason, possesses many attractive features. In this theory the ether and the material ions imbedded in it correspond to the uniform wire and the reactance points considered in this paper. These matters, however, are of a more or less speculative character, and have, therefore, no place here. But it should be noted that the physical problem discussed here was tirst suggested by speculations of this kind. It can be stated as follows: Under what conditions will the non-uniform conductor repre-

1. Reprint from the Transactions of the American Mathematical Society, Vol. I. 
sented in Fig. 3 be approximately equivalent to its corresponding uniform conductor? Or, to be more precise: For what frequencies will an electrical wave have approximately the same wave length and the same damping or attenuation constant on one conductor as on the other?

The mathematical theory developed here gives a definite answer to this question. This answer can be stated in a few words, and for that purpose it is desirable to introduce here a convenient technical term, the so-called angular distance of the interstices - that is, of the intervals between consecutive reactance points. This interval is at any given frequency a definite fraction of the wave length corresponding to that frequency. Let this fraction be $\varphi / 2 \pi$; then $\varphi$ is the angular distance of the interstices. The angular distance of a wave length is, of course, $2 \pi$. The general rule expressing the conditions of equivalence of a non-uniform conductor to its corresponding uniform conductor can now be expressed as follows: For any given frequency a non-uniform conductor of the second type is equivalent to its corresponding uniform conductor as nearly as sin $\varphi / 2$ is to $\varphi / 2$ itself.

The higher the frequency the less resemblance will there be between a given non-uniform conductor and its corresponding uniform conductor. Conversely, if this resernblance is suffciently close for a given frequency, it will be closer for all lower frequencies. When the half-wave length under consideration becomes smaller than the interstices, then the resemblance as far as that wave length and all shorter wave lengths are concerned ceases altogether.

A brief summary of this mathematical paper will be given now.

The main object of this research is the "solution of the problem of Section III. This solution depends on the solution of the problem of Section II, and this again is moulded after the pattern of the solution of the problem of Section I.

In Section I the wave propagation over a uniform wire conductor represented in Fig. 1 is discussed. The effect of the transmitting apparatus $A$ and of the receiving apparatus $B$ is taken into account. Equation [5] is the most general solution of this case. The propagation of waves of both forced and of free periods is easily deduced from it. This particular form of the general solution is new, and it was selected because it is 
best suited for comparing the propagation of waves over nonuniform conductors of Sections II and III to that over their corresponding uniform conductors.

In Section II the wave propagation over a non-uniform conductor of the first type represented in Fig. 2 is discussed. This conductor consists of a certain number of equal coils, $L_{1} \ldots \ldots$. ......... $L_{\mathrm{n}}$, which are connected in series. A certain number of equal condensers, one at each juncture between two consecutive coils, connect this conductor to ground. A transmitting apparatus $A$ and a receiving apparatus $B$ are present. The general solution of this problem, equation (6a), is moulded after the pattern of equation (5) of Section I. Both forced and free oscillations on a conductor of this kind are considered, and the conditions under which it becomes equivalent to its corresponding uniform conductor are worked out at considerable length. The problem of this section and its solution are both new.

The problem of Section III can, from a purely mathematical point of view, be stated as follows: Find the integral of the following partial differential equation :

$$
L \frac{d^{2} y}{d t^{2}}+R \frac{d y}{d t}=\frac{1}{C} \frac{\partial^{2} y}{\partial s^{2}}
$$

and determine it in such a way as to satisfy $k+2$ boundary conditions. Determine also the conditions under which this integral will be equivalent to the integral represented in equation (5), Sec. I.

Equation (2) represents the most general solution, and the constants $x_{1} x_{2} \ldots \ldots \ldots \ldots \ldots x_{\mathrm{k}+2}$ have to be determined from the $k+2$ boundary conditions. The principal mathematical difficulty here reduces itself, then, to the proper mathematical formulation of these boundary conditions so as to obtain a system of equations which can be readily solved. Such a system is system (3) of this section. It is of the same form as system (6) of Section II, the solution of which was obtained in that section. Equations (4) and (5) are thus obtained. Equation (5) is the most general solution, and when this equation becomes very nearly the same as equation (5) of Section $I$, then the nonuniform conductor of this section becomes equivalent to its corresponding uniform conductor. This equivalence cannot be decided without a careful study of the wave lengths and the damping constants of waves of different periods. This study is 
recorded in the remaining portion of this section. This section is also entirely new.

Sec. I and a part of Sec. II, together with a description of experimental investigations bearing upon the same, were published in Vol. XVI of the Transactions of the American Institute of Electrical Engineers for 1899. Additional experimental investigations bearing upon the principal problem-that is, the problem of Sec. III, will be published in the near future.

\section{SECTION I}

WAVE PROPAGATION OVER A UNIFORM LINEAR CONDUCTOR.

\section{A.-Waves of Forced Preiod.}

The conductor is a loop of wire A, B, (Fig. 1.) At one point of the loop is a transmitting apparatus $A$, at the opposite point is a receiving apparatus $\mathrm{B}$. The distance between $\mathrm{A}$ and $\mathrm{B}$ is $l$, equal to one-half the length of the whole loop. The distance of any element $d s$ from $\mathrm{A}$ is $s$.

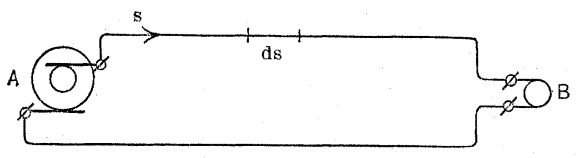

H'TG. 1.

Let $L, R, C$, denote the inductance, resistance and capacity, respectively, per unit length. Let $y$ be the current at any point s. Then

$$
L \frac{d^{2} y}{d t^{2}}+R \frac{d y}{d t}=\frac{1}{C} \frac{\partial^{2} y}{\partial s^{2}}
$$

is the well known equation of propagation.

To formulate the two boundary conditions, one at the receiving and the other at the transmitting apparatus, let $L_{0}, R_{0}, C_{0}$, and $L_{1}, R_{1}, C_{1}$, be the inductance, resistance, and capacity, respectively, of the transmitting and of the receiving apparatus. Each contains a condenser in series with its inductance and resistance. Let the E. M. F. impressed by a be of type $E e^{i p t}$. The boundary equations can now be expressed as follows : 
(2)

$$
\left\{\begin{array}{l}
-2\left(\frac{\partial y}{\partial s}\right)_{s=0}=\left[D_{0}-h_{0} y\right]_{s=0} \text { at transmitting } \\
+2\left(\frac{\partial y}{\partial s}\right)_{s=l}=-\left(h_{1} y\right)_{s=l} \text { at apparatus. }
\end{array}\right.
$$

where

$$
\begin{gathered}
D_{0}=i p C E e^{\mathrm{ipt}} \\
h_{0}=C\left(-p^{2} \lambda_{0}+i p R_{0}\right), h_{1}=C\left(-p^{2} \lambda_{1}+i p R_{1}\right) \\
\lambda_{0}=L_{0}-\frac{1}{p^{2} C_{0}^{2}}, \quad \lambda_{1}=L_{1}-\frac{1}{p^{2} C_{1}}
\end{gathered}
$$

The physical character of the problem suggests the following solution :

$$
y=K_{1} \cos \mu \xi+K_{2} \sin \mu \xi,
$$

where $\xi=l-s$, and $K_{1}$ and $K_{2}$ are proportional to $e^{\text {ipt }}$.

Equation (1) is satisfied for all values of $K_{1}$ and $K_{2}$ if

$$
C\left(-p^{2} L+i p R\right)=-\mu^{2}=-(\alpha+i \beta)^{2}
$$

From this we get

$$
\begin{aligned}
& \alpha=\sqrt{\frac{1}{2} p C\left[\sqrt{p^{2} L^{2}+P^{2}}+p L\right]} \\
& \beta=\sqrt{\frac{1}{2} p C\left[\sqrt{p^{2} L^{2}+R^{2}}-p L\right]}
\end{aligned}
$$

When $p L$ is large in comparison to $R$,

$$
\alpha=p \sqrt{L C}, \quad \beta=\frac{R}{2} \sqrt{\frac{C^{* *}}{L}} .
$$

The boundary equations will be satisfied if

$$
K_{1}=\frac{2 \mu D_{0}}{H^{\prime}}, \quad K_{2}=\frac{h_{1} D_{0}}{H},
$$

where $\quad F=\left(h_{0} h_{1}-4 \mu^{2}\right) \sin \mu l+2 \mu\left(h_{0}+h_{1}\right) \cos \mu l$

* Observe that in the case of a twin conductor where the mutual capacity alone is considered, we must use $2 R$ and $2 L$ in place of $R$ and $L$. 
Hence (3) can now be written

$$
y=\left(2 \mu \cos \mu \xi+h_{1} \sin \mu \xi\right) \frac{D_{0}}{F}
$$

When $h_{0}=h_{1}=0$

$$
y=-\frac{D_{0} \cos \mu \xi}{2 \mu \cdot \sin \mu l}
$$

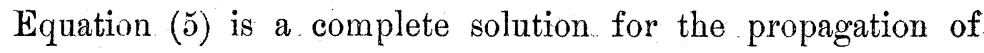
waves of forced period. It represents simple harmonic damped waves. The most essential elements which enter into the description of such waves are the wave length $\lambda$ and the damping or attenuation factor. These can easily be calculated. Since $\mu=\alpha+i \beta$, we shall have $\lambda=2 \pi / \alpha$; the attenuation factor is $e^{-\beta \xi}$

It is evident that $\beta$ diminishes as $L$ increases. A high inductance per unit length means small attenuation and a slow speed of propagation.

\section{B.-Waves W}

Free oscillations are readily calculated for a few special cases. Equation (5) is a general solution for free oscillations also, provided, however, that $\mu$ has such a value as to make

$$
F=0 \text {, since } D_{0}=0 ;
$$

that is, we must have

$$
\left(h_{0} h_{1}-4 \mu^{2}\right) \sin \mu l+2 \mu\left(h_{0}+h_{1}\right) \cos \mu l=0,
$$

but of course, in this case, $h_{0}=k C\left(k \lambda_{0}+R_{0}\right), h_{1}=k C\left(k \lambda_{1}+R\right),-\mu^{2}=k C(k L+R)$.

Equation (6) is a transcendental equation and can be readily solved in a few simple cases.

CASE 1.-The transmitting and receiving apparatus are not present. In this case $h_{0}=h_{1}=0$. Equation (6) reduces to sin $\mu l=0$, therefore $\mu=s \cdot \pi / l$, where $s$ can have any integer value from 1 to $\infty$. The periods of free oscillations are calculated from the equation

$$
-\mu^{2}=k^{2} L C+k R O=-\frac{s^{2} \pi^{2}}{l^{2}}
$$


Therefore

$$
\begin{aligned}
k=-\frac{R}{2 L} & \pm i-1 \\
& \sqrt{\frac{1}{L C} \frac{s^{2} \pi^{2}}{l^{2}}-\frac{R^{2}}{4 L^{2}}} \\
& =-\frac{R}{2 L} \pm i k_{\mathrm{s}} .
\end{aligned}
$$

There are, therefore, an infinite number of periods which are harmonically related to each other unless the damping constant $R / 2 L$ is not negligibly small in comparison with $\pi^{2} / l^{2} L C$. The most general solution of this case can be written

$$
y=e^{-\frac{R t}{2 L}} \sum_{s=1}^{\infty} A_{\mathrm{s}} \cos \left(\frac{s \pi}{l} \xi\right) \cos \left(k_{\mathrm{s}} t-\varepsilon_{\mathrm{s}}\right)
$$

The wave lengths are

$$
\frac{2 l}{1}, \frac{2 l}{2}, \frac{2 l}{3}, \ldots \ldots \ldots \frac{2 l}{s}
$$

CASE 2.-Transmitting apparatus is not present, and in place of the receiving apparatus there is a break in the wire.

In this case $h_{0}=0, h_{1}=\infty$. Equation (6) reduces to

Therefore

$$
\cos \mu l=0 \text {. }
$$

$$
\begin{gathered}
\mu=\frac{2 s+1}{l} \frac{\pi}{2} \\
k=-\frac{R}{2 L} \pm i \sqrt{\frac{1}{L C}\left(\frac{2 s+1}{l} \frac{\pi}{2}\right)^{2}-\frac{R^{2}}{4 L^{2}}} \\
=-\frac{R}{2 L} \pm i k_{2 s+1}, \\
y=e^{-\frac{R t}{2 L}} \sum_{0}^{\infty} A_{2 s+1} \sin \left(\frac{2 s+1}{l} \frac{\pi}{2} \xi\right) \cos \left(k_{2 s+1}-\varepsilon_{2 s+1}\right)
\end{gathered}
$$

The wave lengths are $\frac{4 l}{1}, \frac{4 l}{3}, \frac{4 l}{5}, \ldots \ldots \ldots$

The damping factor is in both cases the same for all frequencies, hence the color of the complex harmonic vibration remains unchanged during the whole epoch of its existence. 


\section{SECTION II. \\ ELECTRICAL OSCILLATIONS ON A NON-UNIFORM CONDUCTOR OF THE} FIRET TYPE.

The conductor consists of $2 n$ equal coils $L_{1}, L_{2} \ldots L_{\mathrm{n}}$ (Fig. 2) connected in series, so as to form a closed loop. At one point $\mathrm{A}$ of this loop is an alternator, at the opposite point is a receiving apparatus, B. At equal distances $2(n-1)$ equal condensers, $C_{1} \ldots \ldots C_{\mathrm{n}-1}$, connect the conductor to ground.

The whole loop is thus divided into $2 n-2$ component circuits $1,2 \ldots \ldots 2 n-2$. It is evident that in the limit when $n$ becomes intinitely large, this conductor becomes an ordinary telegraph or telephone line with uniformly distributed resistance, capacity, and inductance. The question arises now, under what conditions will a conductor of this kind become

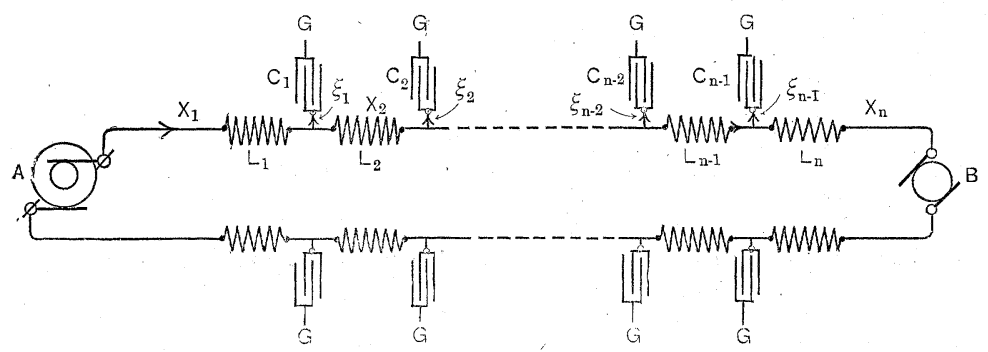

Fig. 2.

equivalent with sufficient approximation to a uniform line, even when $n$ is not infinitely large? This problem does not seem to have been solved before.

In its main features it is similar to that which Lagrange solved in his "Mecanique Analytique, sec. partie, Sec. VI," the problem, namely, of the free vibrations of a weightless string, fixed at its two ends and loaded at equi-distant points by equal weights. But it is much more general, because frictional resistances are taken into consideration, and also because forced as well as free oscillations are considered.

Let $L_{0}, R_{0}, C_{0}$, and $L_{1}, R_{1}$ and $C_{1}$, be the inductance, ohmic resistance, and capacity of $\mathrm{A}$ and $\mathrm{в}$, respectively. Let $L, R, C$, be the corresponding quantities of the coils and condensers in the several component circuits.

The real part of $E e^{\text {ipt }}$ is the E.M.F. impressed by ulternator A. Let $x_{1} \ldots \ldots x_{n}$ be the currents in the component circuits. 
$P_{1} \ldots \ldots P_{n-1}$ are the differences of potential in the line condensers, $C_{1}, C_{2} \ldots \ldots C_{\mathrm{n}-1} ; P_{0}$ and $P^{1}$ are the differences of potential in the condensers in $\mathrm{A}$ and $\mathrm{B}$, respectively $; \xi_{1} \ldots \ldots \xi_{\mathrm{n}-1}$ are the condenser currents. We shall have

$$
\begin{array}{ll}
\xi_{1}=C \frac{d P_{1}}{d t}, & \xi_{2}=C \frac{d P_{2}}{d t}, \ldots \ldots \text { etc. } \\
\xi_{1}=x_{1}-x_{2}, & \xi_{2}=x_{2}-x_{3}, \ldots \text {. etc. }
\end{array}
$$

A.-Forced Oscillations.

Stating the law of equality of action and reaction for each component circuit we obtain the following $n$ differential equations:

$$
\begin{aligned}
& \left(L_{0}+2 L\right) \frac{d x_{1}}{d t}+\left(R_{0}+2 R\right) x_{1}+2 P_{1}+P_{0}=E e^{\mathrm{pt} t} \\
& L \frac{d x_{2}}{d t}+R x_{2}+P_{2}-P_{1}=0 \\
& \left(L_{1}+2 L\right) \frac{d x_{\mathrm{n}}}{d t}+\left(R_{1}+2 R\right) x_{\mathrm{n}}-2 P_{\mathrm{n}-1}+P^{1}=0
\end{aligned}
$$

When the steady state has been reached, the currents will be just like the impressed .. M. F., simple harmonics of the time $t$, that is,

$$
x_{1}=A_{1} e^{\mathrm{ipt}}, \quad x_{2}=A_{2} e^{\mathrm{ipt}},
$$

where $\mathrm{A}_{1}, A_{2} \ldots \ldots$ are complex quantities.

Differentiating each member of (2) and substituting from (3) and (1), we obtain

$$
\begin{aligned}
h x_{1}+\xi_{1}-0 & =D, \\
h x_{2}+\xi_{2}-\xi_{1} & =0, \\
\ldots \ldots \ldots \ldots \ldots \ldots \ldots \ldots \ldots \ldots & \ldots \ldots \\
h x_{\mathrm{n}-1}+\xi_{\mathrm{n}-1}-\xi_{\mathrm{n}-2} & =0, \\
h x_{\mathrm{n}}+0-\xi_{\mathrm{n}-1} & =-h_{1} x_{\mathrm{n}},
\end{aligned}
$$


where

$$
h=C\left(-p^{2} L+i p R\right)
$$

$$
\begin{gathered}
D=\frac{1}{2} i p C E e^{\mathrm{tpt}}-\frac{1}{2} C\left(-p^{2} \lambda_{0}+i p R_{0}\right)=D_{0}-h_{0} x_{1}, \\
h_{1}=\frac{1}{2} C\left[-p^{2} \lambda_{1}+i p R_{1}\right], \\
\lambda_{0}=L_{0}-\frac{1}{p^{2} C_{0}}, \quad \lambda_{1}=L_{1}-\frac{1}{p^{2} C_{1}} .
\end{gathered}
$$

Another form is obtained by substituting for $\xi_{1}, \xi_{2} \ldots \ldots$ as follows :

$$
\begin{aligned}
& (h+1) x_{1}+0-x_{2}=D=D_{0}-h_{0} x_{1} \\
& (h+2) x_{2}-x_{1}-x_{3}=0 \\
& \cdots \ldots \ldots \ldots \ldots \ldots \cdots \cdots \cdots \cdots \cdots \cdots \cdots \\
& (h+2) x_{\mathrm{n}-1}-x_{\mathrm{n}-2}-x_{\mathrm{n}}=0 \\
& (h+1) x_{\mathrm{n}}-x_{\mathrm{n}-1}-0=-h_{1} x_{\mathrm{n}} .
\end{aligned}
$$

Equation (3) of Sec. 1 suggests the following solution:

$$
x_{\mathrm{m}}=K_{1} \cos 2(n-m) \theta+K_{2} \sin 2(n-m) \theta[6 \mathrm{a}]
$$

If $h+2=2 \cos 2 \theta$ then all the equations except the first and the last will be satisfied for all values of $K_{1}$ and $K_{2}$. These two equations, which correspond to the boundary equations of Sec. 1 ., will be also satisfied if we assign to $K_{1}$ and $K_{2}$ suitable values, as follows :

$$
K_{2}=\frac{\left(h_{1}-1\right)+\cos 2 \dot{\theta}}{\sin 2 \theta} \quad K_{1}
$$

$K_{1}=\frac{D_{0} \sin 2 \theta}{h_{0} h_{1} \sin 2(n-1) \theta-4 \sin ^{2} \theta \sin 2 n \theta+2\left(h_{0}+h_{1}\right) \sin \theta \cos (2 n-1) \theta}$

we can now write,

$x_{\mathrm{m}}=\frac{\left[2 \sin \theta \cos (2 n-2 m-+1) \theta+h_{1} \sin 2(n-m) \theta\right] D_{0}}{h_{0} h_{1} \sin 2(n-1) \theta-4 \sin \theta \sin 2 n \theta+2\left(h_{0}+h_{1}\right) \sin \theta \cos (2 n-1) \theta}$

$\theta$ is a complex angle, hence the forced oscillations of type $e^{\mathrm{ipt}}$ on a non-uniform conductor of this kind are simple harmonic damped oscillations. The similarity between this conductor and a uniform wire will be discussed presently. When $h_{0}=h_{1}=0$ we obtain

$$
x_{\mathrm{m}}=\frac{-I_{0} \cos (2 n-2 m+1) \theta}{2 \sin \theta \sin 2 n \theta}
$$




\section{B.-Free Oscillations.}

Equation (7) holds true for free as well as for forced oscillations. But since in the case of free oscillations $D_{0}=o$ it follows that the denominator of $(7)$ must vanish to prevent the vanishing of all currents. We shall have, therefore,

$$
\begin{aligned}
& h_{0} h_{1} \sin (2 n-2) \theta-4 \sin ^{2} \theta \sin 2 n \theta+ \\
& +2\left(h_{0}+h_{1}\right) \sin \theta \cos (2 n-1) \theta=o
\end{aligned}
$$

From this equation $\theta$ and the corresponding periods and damping constants have to be determined. A solution can be readily obtained for a small number of cases. The two most important will be considered here.

First.-The transmitting and the receiving apparatus are not present. In this case $h_{0}=h_{1}=0$, and

$$
x_{\mathrm{m}}=B \cos (2 n-2 m+1) \theta
$$

It is found from (8) that (9) is actually the solution of the differential equations (6) for $h_{0}=h_{1}=D_{0}=0$, provided that $\theta=\frac{s \pi}{2 n}$, where $s$ is any integer from 1 to $2 n$.

Hence the most general solution will be

$$
x_{\mathrm{m}}=\sum_{s=1}^{2 n} B_{\mathrm{s}} \cos (2 n-2 m+1) \frac{s \pi}{2 n}
$$

But it should be observed now that $x_{\mathrm{m}}$ is a periodic function of the time, that is

$$
x_{\mathrm{m}}=\sum_{s=1}^{\infty} K_{\mathrm{s}} e^{p_{\mathrm{s}} t}
$$

Hence in (10) each amplitude $B$ contains the time factor $e^{\mathrm{pt}}$.

The constant $p_{\mathrm{s}}$ which measures the period and the damping constant of the free oscillation is determined from the relation:

$$
h=-4 \sin ^{2} \theta .
$$

In the case of free oscillations

$$
h=p_{\mathrm{s}}{ }^{2} L C+p_{\mathrm{s}} R C, \theta=\frac{s \pi}{2 n} .
$$


Hence

$$
p_{\mathrm{s}}^{2} L C+p_{\mathrm{s}} R C=-4 \sin ^{2} \frac{s \pi}{2 n} .
$$

Before solving this equation, it is desirable to make the following substitution:

Let $L,{ }^{\prime} C,^{\prime} R^{\prime}$ be the total inductance, capacity, and resistance, respectively, of one-half of the conductor, then

$$
L=\frac{L^{\prime}}{n}, C=\frac{C^{\prime}}{n}, R=\frac{R^{\prime}}{n} .
$$

Let $l$ denote the half length of a uniform wire having $\sigma, r, c$, for inductance, resistance, and capacity per unit length, and let it have the same total inductance, resistance, and capacity as the non-uniform conductor. Since

$$
l \sigma=L^{\prime}, l r=R^{\prime}, l_{c}=C^{\prime},
$$

we shall have

$$
L=\frac{l \sigma}{n}, C=\frac{l c}{n}, R=\frac{l r}{n} .
$$

This uniform wire having the same total inductance, resistance, and capacity as the non-uniform conductor, will be called the "corresponding uniform conductor."

From (11) we obtain

$$
\frac{l^{2}}{n^{2}}\left(p_{\mathrm{s}}^{2} \sigma c+p_{\mathrm{s}} c r\right)=-4 \sin ^{2} \frac{s \pi}{2 n},
$$

therefore

$$
\begin{gathered}
p_{\mathrm{s}}=-\frac{r}{2 \sigma} \pm \sqrt{-1} \sqrt{\frac{1}{\sigma c} \frac{4 n^{2}}{l^{2}} \sin ^{2} \frac{8 \pi}{2 n}-\frac{r^{2}}{4 \sigma^{2}}}, \\
p_{\mathrm{s}}=\frac{r}{2 \sigma} \pm i k_{\mathrm{s}}
\end{gathered}
$$

Equation (10) becomes now :

$$
x_{\mathrm{m}}=e^{\frac{-r t}{2 \sigma} \sum_{1}^{2 n}} A_{\mathrm{s}} \cos (2 n-2 m+1) \frac{s \pi}{2 n} \cos \left(k_{\mathrm{s}} t-\varepsilon_{\mathrm{s}}\right) .
$$


It is clear that the oscillations on the non-uniform conductor of type (1) have the same damping constant as the oscillations on the corresponding uniform conductor. It will be shown presently that under eertain conditions they will also have the longer periods up to a certain limit very nearly the same as the corresponding uniform conductor. For these periods, then, the nonuniform conductor will be equivalent to its corresponding uniform conductor.

Second Case.-The transmitting apparatus is not present, and in place of the receiving apparatus there is a break in the line at $B$.

In this case $h_{0}=0, h_{1}=\infty$. Equation (9) gives

$$
x_{\mathrm{m}}=B \sin (2 n-m+2) \theta \text {, }
$$

provided that

$$
\cos (2 n-1) \theta=0
$$

or

$$
\theta=\frac{2 s+1}{2 n-1} \frac{\pi}{2} .
$$

We shall have, therefore,

$$
\begin{gathered}
p_{2 \mathrm{~s}+1}=\frac{-r}{2 \sigma} \pm i \sqrt{\frac{1}{\sigma c} \frac{4 n^{2}}{l^{2}} \sin ^{2} \frac{2 s+1}{2 n-1} \frac{\pi}{2}-\frac{r^{2}}{4 \sigma^{2}}} \\
=-\frac{r}{2 \sigma} \pm i k_{2 \mathrm{~s}+1}
\end{gathered}
$$

Hence

$$
\begin{array}{r}
x_{\mathrm{m}}=e^{-\frac{r t}{2 \sigma}} \sum_{s}^{2 n} A_{2 \mathrm{~s}+1} \sin (2 n-2 m+2) \frac{2 s+1}{2 n-1} \frac{\pi}{2} \\
\cos \left(k_{2 \mathrm{~s}+1} t-\varepsilon_{2 \mathrm{~s}+1}\right) .
\end{array}
$$

The same remark regarding the damping constant and the free periods applies here as in the preceding case.

\section{The Wave Lengths of Free Oscillations.}

The angles $s \pi / 2 n$ and $(2 s+1) \pi / 2(2 n-1)$ have an interesting physical meaning which will be brought out by consid- 
ering the wave-lengths of free oscillations. Consider one of the component harmonics of $x_{\mathrm{m}}$ in Case I, say

$$
\xi_{\mathrm{m}, \mathrm{s}}=A_{\mathrm{s}} \cos (2 n-2 m+1) s \pi / 2 n \cos \left(k_{\mathrm{s}} t-\varepsilon_{\mathrm{s}}\right) .
$$

Compare it to the corresponding component of $x_{\mathrm{m}_{1}}$, that is to

$$
\xi_{\mathrm{m}_{1}, \mathrm{~s}}=A_{\mathrm{s}} \cos \left(2 n-2 m_{1}+1\right) s \pi / 2 n \cos \left(k_{\mathrm{s}} t-\varepsilon_{\mathrm{s}}\right) .
$$

If they are a wave length apart, $\xi_{\mathrm{m}, \mathrm{s}}=\xi_{\mathrm{m}_{1}, \mathrm{~s}}$ and $m_{1}-m=$ number of coils covered by one wave length. But in this case

$$
(2 n-2 m+1) s \pi / 2 n=\left(2 n-2 m_{1}+1\right) s \pi_{/}^{\prime} 2 n+2 \pi,
$$
therefore

$$
m_{1}-m=2 n / \mathrm{s}=\nu_{\mathrm{s}} \text {. }
$$

$\nu_{\mathrm{s}}=2 \mathrm{n} / \mathrm{s}$ is, therefore, the number of coils covered by a wave length which corresponds to the harmonic $s$. It can be shown that in the second case

$$
\frac{2(2 n-1)}{2 s+1}=\nu_{s}
$$

For $\frac{s \pi}{2 n}$ and $\frac{2 s+1}{2 n-1} \frac{\pi}{2}$ we can, therefore, write $\frac{\pi}{\nu_{\mathrm{s}}}=\frac{1}{2} \frac{2 \pi}{\nu_{\mathrm{s}}}$

The physical meaning of $2 \pi / \nu_{\mathrm{s}}$ can now be readily fixed. A coil represents a definite fraction of a wave length, and this fraction will have a different value for different harmonics. The higher the harmonic the shorter will be the wave length, and therefore the larger will be the value of this fraction. It is convenient, however, to measure this fraction in terms of an angle instead of in terms of a ware length. If we arbitrarily assume that an angular distance $2 \pi$ corresponds to a wave length, then an angular distance $2 \pi / n$ will correspond to the $n$th part of a wave length. With this understanding

$$
\frac{s \pi}{2 n} \text { and } \frac{2 s+1}{2 n-1} \frac{\pi}{2}, \text { that is, } \frac{1}{2} \frac{2 \pi}{\nu_{s}}
$$

represents one-half of the angular distance covered by a coil.

A Non-Uniform Conductor of the First Type Compared to its Corresponding Uniform Wire.

a.-Similarity with Respect to Free Oscillations.

Comparing the expressions for the free periods of oscillation 
which were obtained in Sections I and II we see that as long as $\pi / \nu_{\mathrm{s}}$ can be written for sin $\pi / \nu_{\mathrm{s}}$ so long will the periods of free oscillations of the non-uniform conductor be nearly the same as those of its corresponding uniform conductor. We have, therefore, the simple rule: A non-uniform conductor of the first type represents its corresponding uniform conductor as nearly as one-half of the angular distance covered by one of its coils represents the sine of that distance. The non-uniform conductor employed by $\mathrm{me}^{1}$ in my experiments had 400 coils. In this case

$$
\frac{\pi}{\nu_{s}}=\frac{s \pi}{400}
$$

For $s=25$ we have $\pi \nu_{s}=\pi / 16$. Now, $\sin \pi / 16$ differs from $\pi / 16$ by $\frac{2}{3}$ of one per cent. of the value of $\pi / 16$. Hence, the period of the 25th harmonic of my non-uniform conductor differs from that of the same harmonic on the corresponding uniform wire by less than $\frac{\dot{\alpha}}{3}$ of one per cent. For lower harmonics the difference is smaller. The 25th harmonic had approximately 3500 P.P.s. Hence. up to the 25 th harmonic-that is, up to 3500 P.P.s. - the non-uniform conductor employed by me had nearly the same free periods as its corresponding uniform conductor.

\section{b.--Similarity With Respect to Forced Oscillations.}

The wave length and the attenuation constant corresponding to a given frequency speed $p_{\mathrm{s}}$ can be studied by studying the angle $\theta$ from the following equation :

$$
\begin{aligned}
& -4 \sin { }^{2} \theta_{\mathrm{s}}=-p_{\mathrm{s}}^{2} L C+i p_{\mathrm{s}} R C=\frac{l^{2}}{n^{2}}\left[-p_{\mathrm{s}}^{2} \sigma C+i p_{\mathrm{s}} r C\right] \\
& =-\frac{l^{2}}{n^{2}} \mu_{\mathrm{s}}^{2}=-\frac{l^{2}}{n^{2}}\left(\alpha_{\mathrm{s}}+i \beta_{\mathrm{s}}\right)^{2} \text { or } \sin \theta_{\mathrm{s}}=\frac{1}{2} \frac{l}{n}\left(\alpha_{\mathrm{s}}+i \beta_{\mathrm{s}}\right)
\end{aligned}
$$

where $\sigma, c, r$ are the inductance, eapacity and resistance, respectively, per unit length of the corresponding uniform conductor; $\mu_{\mathrm{s}}, \alpha_{\mathrm{s}}$ and $\beta_{\mathrm{s}}$ have the same meaning as in Sec. I.

Referring now to equation (4) Sec. I, it will be found that

$$
\alpha_{\mathrm{s}} \geq \beta_{\mathrm{s}} \text {. }
$$

1. See paper cited in the introduction. 
If, therefore, $\frac{1}{2} \alpha_{\mathrm{s}} / 2 n$ is sufficiently small then we can put

$$
\theta_{\mathrm{s}}=\frac{1}{2} \frac{l}{n}\left(\alpha_{\mathrm{s}}+i \beta_{\mathrm{s}}\right)=\frac{1}{2} \frac{l}{n} \mu_{\mathrm{s}}
$$

When this substitution is made in ( 7$)$ and $\left(7^{a}\right)$ these equations will transform into (5) and $\left(5^{a}\right)$ of Sec. I, which shows that under these conditions the non-uniform conductor of the first type becomes approximately equivalent to its corresponding uniform conductor, the degree of approximation being the same as the degree of equality between $\sin \theta_{\mathrm{s}}$ and $\theta_{\mathrm{s}}$.

The physical meaning of this ean be readily made clear. If by $\lambda_{s}$ we denote the wave length on the corresponding uniform conductor corresponding to the frequency speed $p_{\mathrm{s}}$, then according to Sec. I,

$$
\alpha_{\mathrm{s}}=\frac{2 \pi}{\lambda_{\mathrm{s}}}, \frac{l}{n} \alpha_{\mathrm{s}}=\frac{l}{n} \frac{2 \pi}{\lambda_{\mathrm{s}}}
$$

Let $n_{\mathrm{s}}$ be the number of coils on the non-uniform conductor which cover a wave length of frequency speed $p_{\mathrm{s}}$, and let $\varphi_{\mathrm{s}}$ be the angular distance covered by a coil, then

$$
\varphi_{\mathrm{s}}=\frac{2 \pi}{n_{\mathrm{s}}}
$$

Again

$$
\begin{gathered}
n: n_{\mathrm{s}}:: l: \lambda_{\mathrm{s}}, \\
n: \frac{2 \pi}{\varphi_{\mathrm{s}}}:: l: \lambda_{\mathrm{s}} .
\end{gathered}
$$

or

Hence

$$
\frac{l}{n} \frac{2 \pi}{\lambda_{\mathrm{s}}}=\frac{l}{n} \alpha_{\mathrm{s}}=\varphi_{\mathrm{s}}
$$

The substitution mentioned above will therefore be permissible when $\frac{1}{2} \varphi_{\mathrm{s}}$ (that is, one-half of the angular distance covered by a coil) is approximately equal to $\sin \varphi_{\mathrm{s}} / 2$.

We have here the same rule as in the case of free oscillations. It will be shown in the next section that this rule is also applicable to non-uniform conductors of the second type for waves of both forced and of free periods. 
SECTION III.

WAVE PROPAGATION OVER A NON-UNIFORM CONDUCTOR OF THE SECOND TYPE.

\section{A. Waves of Forced Period.}

Such a conductor is represented in diagram of Fig. 3. A long, uniform wire forms a loop $L_{1} \ldots L_{k+2}$. At equal intervals are inserted in series $2 k+2$ equal coils $L_{1} L_{2} \ldots$. To make the discussion more general, let each coil have a condenser of capacity $C_{0}$ in series with it.

Let $L, R, C$ represent the inductance, resistance, and capacity, respectively, per unit length of the uniform wire. Let an E. M. F. of type $E e^{\text {ipt }}$ be impressed at $L$. Employing the usual notation we shall have the following equation of propagation at every point of the uniform wire:

$$
L \frac{d^{2} y}{d t^{2}}+R \frac{d y}{d t}=\frac{1}{C} \frac{\partial^{2} y}{\partial s^{2}}
$$

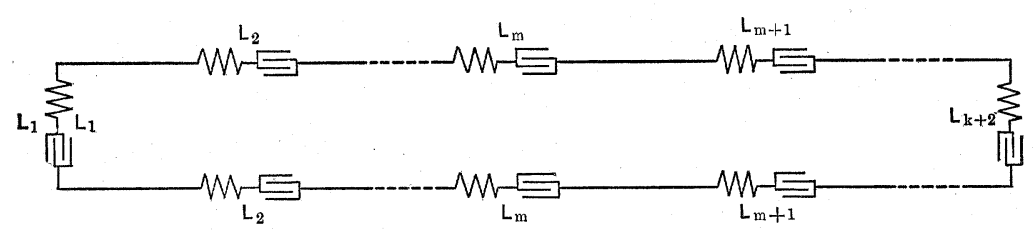

Fig. 3.

The same as in Sec. I, but here the integral has to satisfy $2 \mathrm{k}+2$ boundary conditions, one at each coil. On account of the symmetry of distribution of the coils on the two sides of the loop this number is reduced to $\mathrm{k}+2$.

To formulate these boundary conditions introduce the following notation:

Let $2 l=$ length of loop; $l /(k+1)$ will be the distance between two consecutive coils. Let $L_{0}, K_{0}, C_{0}$ be the inductance, resistance and capacity, respectively, of each coil. Let $y_{\mathrm{m}}$ be the current at any point of the interval between coils $L_{\mathrm{m}}$ and $L_{\mathrm{m}+1}$. Denote the distance of this point from coil $L_{\mathrm{m}}$ by $\xi$.

The currents in the coils will be denoted by $x_{1} x_{2} \quad x_{\mathrm{k}+1} x_{\mathrm{k}+2}$ It is evident that

$$
\left(y_{\mathrm{m}}\right)_{\xi=0}=\left(y_{\mathrm{m}-1}\right)_{\xi=l / k+1}=x_{\mathrm{m}} .
$$


The boundary conditions for all coils except $L_{1}$ and $L_{\mathrm{k}+2}$ can now be stated.

$$
\begin{gathered}
\left(\frac{\partial y_{\mathrm{m}-1}}{\partial \xi}\right) \xi_{=l / k+1}-\left(\frac{\partial y_{\mathrm{m}}}{\partial \xi}\right) \xi_{=0}= \\
=-C\left(-p^{2} \lambda_{0}+i p R_{0}\right) x_{\mathrm{m}}=-h x_{\mathrm{m}},
\end{gathered}
$$

where

$$
\lambda_{0}=L_{0}-\frac{1}{p^{2} C_{0}^{\gamma}} \text {. }
$$

There are $k$ equations of this form. The boundary equations for the first and the last coil are of the following form:

$$
-2\left(\frac{\partial y_{1}}{\partial \xi}\right)_{\xi=0}=i \cdot p C E e^{\mathrm{ipt}}-h x_{1}=D-h x_{1}
$$

for the first coil, and

$$
2\left(\frac{\partial y_{\mathrm{k}+1}}{\partial \xi}\right)_{\xi=l / k+1}=-h x_{\mathrm{k}+2}
$$

for the last coil.

Equation (3) of Sec. I and equation (6a) of Sec. II suggest the following solution:

$$
y_{\mathrm{m}}=K_{1} \cos \mu \xi+K_{2} \sin \mu \xi
$$

where $\mu$ has the same value as in Sec. I.

When $\xi=0, y_{\mathrm{m}}$ becomes $x_{\mathrm{m}}$, and when $\xi=l / k+1, y_{\mathrm{m}}$ becomes $x_{\mathrm{m}+1}$.

Hence

$$
x_{\mathrm{m}}=K_{1}, x_{\mathrm{m}+1}=K_{1} \cos \frac{\mu l}{k+1}+K_{2} \sin \frac{\mu l}{k+1} .
$$

Therefore

$$
y_{\mathrm{m}}=\frac{x_{\mathrm{m}} \sin \mu\left(\frac{l}{k+1}-\xi\right)+x_{\mathrm{m}+1} \sin \mu \xi}{\sin \frac{\mu l}{k+1}}
$$

We have now to determine the $\mathrm{k}+2$ constants $x_{1} x_{2} \ldots x_{\mathrm{k}+2}$ 
from the $k+2$ boundary equations. Begin with the boundary equation at the coil $L_{1}$ :

$$
\frac{\partial y_{\mathrm{m}}}{\partial \xi}=\frac{\mu\left[-x_{\mathrm{m}} \cos \mu\left(\frac{l}{k+1}-\xi\right)+x_{\mathrm{m}+1} \cos \mu \xi\right]}{\sin \frac{\mu l}{k+1}}
$$

Therefore

$$
\begin{gathered}
-2\left(\frac{\partial y_{1}}{\partial \xi}\right)_{\xi=0}=2\left(x_{1} \cos \frac{\mu l}{k+1}-x_{2}\right) \frac{\mu}{\sin \frac{\mu l}{k+1}}=D-h_{\nu} x \\
\text { Let } \sigma=h \frac{\sin \frac{\mu l}{k+1}-4 \sin ^{2} \frac{1}{2} \frac{\mu l}{k+1},}{\mu}=\frac{\sin \frac{\mu l}{k+1}}{\mu} D ;
\end{gathered}
$$

This boundary equation can then be written:

$$
(\sigma+1) x_{1}-0-x_{2}=\frac{\rho}{2}+\frac{\sigma}{2} x_{1}
$$

Similarly $(\sigma+2) x_{2}-x_{1}-x_{3}=0$

$$
\begin{aligned}
& (\sigma+2) x_{3}-x_{2}-x_{4}=0 \\
& \ldots \ldots \ldots \ldots \ldots \ldots \ldots \\
& (\sigma+2) x_{\mathrm{m}}-x_{\mathrm{m}-1}-x_{\mathrm{m}+1}=0 \\
& \ldots \ldots \ldots \ldots \ldots \ldots \ldots \\
& (\sigma+2) x_{\mathrm{k}+1}-x_{\mathrm{k}}-x_{\mathrm{k}+2}=0 \\
& (\sigma+1) x_{\mathrm{k}+2}-x_{\mathrm{k}+1}-0=\frac{\sigma}{2} x_{\mathrm{k}+2}
\end{aligned}
$$

This system is of the same form as system (6) of Sec. II. Equation (7) of that section enables us to write down the solution of (3). 
Let

$$
\sigma+2=2 \cos 2 \psi
$$

then

$$
x_{\mathrm{m}}=-\frac{D \sin \frac{\mu l}{k+1} \cos 2(k-m+2) \psi}{2 \mu \sin 2 \phi \sin 2(k+1) \phi}
$$

$$
y_{\mathrm{m}}=\frac{\left[\cos 2(k-m+2) \psi \sin \mu\left(\frac{l}{k+1}-\xi\right)+\cos 2(k-m+1) \psi \sin \mu \xi\right] D}{2 \mu \sin 2 \phi \sin 2(k+1) \psi}
$$

These equations give the complete mathematical representation of waves of forced period on a conductor of this kind. $\varphi$ and $\mu$ are, of course, complex angles; hence, in order to calculate from these equations in any particular case the principal quantities with which we are concerned in wave propagation, namely, the wave length and the attenuation constant, it would be necessary to study separately the real and the imaginary parts of these equations. This study in its broadest aspect presents considerable mathematical complexity. It is, however, somewhat remote from the principal aim of this paper, which is to ascertain the conditions under which a non-uniform conductor of the second type is approximately equivalent to its corresponding uniform conductor; that is to say, for what frequencies will the two have approximately the same wave lengths and the same attenuation constants?

Equivalence of a Non-Uniform Conductor of the Second Type to its Corresponding Uniform Conductor.

An answer to this question is obtained by studying the follow ing equation:

$$
\frac{h_{\mathrm{s}} \sin \frac{\mu_{\mathrm{s}} l}{k+1}}{\mu_{\mathrm{s}}}+2 \cos \frac{\mu_{\mathrm{s}} l}{k+1}=2 \cos 2 \psi_{\mathrm{s}},
$$

or

$$
\frac{h_{\mathrm{s}} \sin \frac{\mu_{\mathrm{s}} l}{k+1}}{\mu_{\mathrm{s}}}-4 \sin ^{2} \frac{1}{2} \frac{\mu_{\mathrm{s}} l}{k+1}=-4 \sin ^{2} \psi_{\mathrm{s}}
$$

The subscript $s$ denotes that the particular frequency speed $p_{\mathbf{s}}$ s considered.

$$
\mu_{\mathrm{s}}=\alpha_{\mathrm{s}}+i \beta_{\mathrm{s}}, \quad \alpha_{\mathrm{s}} \geq \beta_{\mathrm{s}} .
$$


If $\lambda_{\mathrm{s}}$ is the wave length corresponding to $p_{\mathrm{s}}$ on the uniform wire before the introduction of the reactance points, then

$$
\frac{l}{k+1} \alpha_{\mathrm{s}}=\frac{l}{k+1} \frac{2 \pi}{\lambda_{\mathrm{s}}}
$$

Let $\theta_{\mathrm{s}}$ be the angular distance between two consecutive coils for wave length $\lambda_{s}$, then

$$
\begin{aligned}
2 \pi: \lambda_{\mathrm{s}}:: \theta_{\mathrm{s}}: \frac{l}{k+1} & \theta_{\mathrm{s}}=\frac{l}{k+1} \frac{2 \pi}{\lambda_{\mathrm{s}}}=\frac{l}{k+1} \alpha_{\mathrm{s}}
\end{aligned}
$$

Hence, if $\theta_{\mathrm{s}}$ is so small that $\sin \theta_{\mathrm{s}}=\theta_{\mathrm{s}}$ very nearly, then

$$
\frac{l}{k+1} h_{\mathrm{s}}-\left(\frac{l}{k+1}\right)^{2} \mu_{\mathrm{s}}^{2}=-4 \sin ^{2} \phi_{\mathrm{s}}
$$

or

$$
\left(\frac{l}{k+1}\right)^{2} C\left[-p_{\mathrm{s}}^{2}(\rho+L)+i p(r+R)\right]=-4 \sin ^{2} \psi_{\mathrm{s}}
$$

where $\rho$ and $r$ are the inductance and resistance, respectively, per unit length of the inserted coils, so that $\rho+L$ and $r+R$ are the inductance and resistance, respectively, of the corresponding uniform conductor. This last equation can also be written :

$$
\frac{1}{2} \frac{l}{k+1}\left(\alpha_{\mathrm{s}}{ }^{1}+i \beta_{\mathrm{s}}{ }^{1}\right)=\sin \psi_{\mathrm{s}}
$$

In this case also $\alpha_{\mathrm{s}}{ }^{1} \geq \beta_{\mathrm{s}}{ }^{1}$. Let $\lambda_{\mathrm{s}}{ }^{1}$ be the wave length for frequency speed $p_{\mathrm{s}}$ on the corresponding uniform conductor, then

$$
\alpha_{\mathrm{s}}^{1}=\frac{2 \pi}{\lambda_{\mathrm{s}}{ }^{1}} \text {. }
$$

Put $\varphi_{\mathrm{s}}{ }^{1}=l \alpha_{\mathrm{s}}{ }^{1} /(k+1)$ then $\varphi_{\mathrm{s}}{ }^{1}$ is the angular distance between two consecutive coils, the angular distance of $\lambda_{\mathrm{s}}{ }^{1}$ being $2 \pi$. then

If, therefore, $\frac{1}{2} \varphi_{\mathrm{s}}{ }^{1}$ is so small that $\sin \frac{1}{2} \varphi_{\mathrm{s}}{ }^{1}=\frac{1}{2} \varphi_{\mathrm{s}}{ }^{1}$ very nearly, very nearly.

$$
\phi_{\mathrm{s}}=\frac{1}{2} \frac{l}{h+1}\left(\alpha_{s}{ }^{1}+i \beta_{s}{ }^{1}\right)=\frac{1}{2} \frac{l}{k+1} \mu_{s}{ }^{1},
$$


Making this substitution in (4) we obtain equation (5a), Sec. I. The equation of wave propagation for a non-uniform conductor of the second type is under these conditions the same as that for its corresponding uniform conductor. The two will have approximately the same wave length and the same attenuation constant for the frequency speed $p_{\mathrm{s}}$. The degree of approximation is the same as the degree of approximation in the equality between

$$
\sin \varphi_{\mathrm{s}} / 2 \text { and } \varphi_{\mathrm{s}} / 2 \text {. }
$$

A numerical example will illustrate this point more clearly.

Consider a uniform telegraph wire having the following constants per mile:

$$
\begin{aligned}
& L=.004 \text { henrys } \\
& R=7 \text { ohms } \\
& C=.01 \times 10^{-6} \text { farads }
\end{aligned}
$$

Introduce at each mile a coil having

$$
\begin{aligned}
& L_{1}=.036 \text { henrys } \\
& R_{1}=1 \mathrm{ohm}
\end{aligned}
$$

The non-uniform conductor thus obtained will have for its corresponding uniform conductor a uniform wire having per mile

$$
\begin{aligned}
L+L_{1} & =.04 \text { henrys } \\
R+R_{1} & =8 \mathrm{ohms} \\
C & =.01 \times 10^{-6} \text { farads }
\end{aligned}
$$

For a frequency of 1500 P.P.s. we shall have $p_{\mathrm{s}}=2 \pi \times 1500$. The wave length $\lambda_{8}{ }^{1}$ on this corresponding uniform conductor for frequency of 1500 P.P.s. is very nearly

$$
\lambda_{\mathrm{s}}{ }^{1}=33 \text { miles. }
$$

Hence, since the interposed coils are one mile apart we shall have

$$
\sin \frac{1}{2} \varphi^{1}=\sin \pi / 33
$$

Now, $\sin \pi / 33=\pi / 33$ to within one-sixth of one per cent. of the value of $\pi / 33$. Up to this degree of approximation will, then, the non-uniform conductor just described have the same wave length and the same attenuation constant as its correspond- 
ing uniform conductor. For lower frequencies the degree of approximation will be much higher; hence, for all frequencies which are of any importance in the telephonic transmission of speech the two conductors are equivalent.

\section{B. Waves of Free Perion.}

Equation (4) holds for all values of $D$; it should therefore hold good when $D=O$. In this case, however, all the currents will vanish unless the denominator vanishes-that is, unless

$$
\sin 2 \phi \sin 2(\mathrm{k}+1) \psi=0 \ldots \ldots\left(4^{\mathrm{a}}\right) .
$$

This transcendental equation, together with the equation

$$
\frac{h}{\mu} \sin \frac{\mu l}{k+1}+2 \cos \frac{\mu l}{k+1}=2 \cos 2 \psi
$$

determines the free periods and their corresponding damping constants.

Equation (4a) will be fulfilled when

$$
\phi=\frac{r \pi}{2(k+1)}
$$

where $r$ is any integer from 0 to $\infty$.

When $r=s(\mathrm{k}+1)$ then we shall have not only

$$
\sin 2(\mathrm{k}+1) \psi=0 \text {, but also } \sin 2 \psi=0 .
$$

These particular values of $\psi$ give a set of free oscillations which form a distinct group, called further below the oscillations of normal period.

Equation (4b) should, therefore, be written :

$$
\frac{h_{\mathrm{r}} \sin \frac{\mu_{\mathrm{r}} l}{k+1}}{\mu_{\mathrm{r}}}+2 \cos \frac{\mu_{\mathrm{r}} l}{k+1}=2 \cos \frac{r \pi}{k+1}
$$

This equation will be referred to as the period equation. It should be observed that

$$
-\mu_{\mathrm{r}}^{2}=C\left(\nu_{\mathrm{r}}^{2} L+\nu_{\mathrm{r}} R\right), \quad h_{\mathrm{r}}=C\left(\nu_{\mathrm{r}}^{2} \dot{\lambda}_{0}+\nu_{\mathrm{r}} R_{0}\right)
$$


An inspection of (5) shows that the complete solution for waves of free period can now be written:

$$
\begin{aligned}
& y_{\mathrm{m}}=\sum_{r=1}^{\infty} \frac{A_{\mathrm{r}}}{\sin \frac{r \pi}{k+1}}\left\{\cos (k-m+2) \frac{r \pi}{k+1} \sin \mu_{\mathrm{r}} \frac{l}{k+1}(-\xi)+\right. \\
& \left.\cos (k-m+1) \frac{r \pi}{k+1} \sin \mu_{\mathrm{r}} \xi\right\}=\sum_{r=1}^{\infty} \eta_{\mathrm{r}}
\end{aligned}
$$

In the case of a uniform wire the periods and the wave lengths of the simple harmonic components $\eta_{1} \eta_{2} \ldots . \eta_{r} \ldots$. have in general a harmonic relation. The introduction of the reactance points $L_{1} L_{2} \ldots$. disturbs this relation in consequence of a displacement in the values of the normal periods and of the normal damping constant. It is evident from purely physical considerations that since the introduction of the reactance points increases in general the inductance per unit length the periods will be lengthened.

a. Waves of Normal Period.

According to equation (7), Sec. I, the wave lengths of free oscillations on a uniform loop are as follows:

$$
\begin{gathered}
\frac{2 l}{1} \frac{2 l}{2}, \frac{2 l}{3}, \ldots \frac{2 l}{k+1}, \frac{2 l}{k+2}, \cdots \frac{2 l}{2(k+1)}, \frac{2 l}{2(k+3)}, \\
\ldots \frac{2 l}{s(k+1)}, \frac{2 l}{s(k+1)+1} \cdot \cdots
\end{gathered}
$$

Divide this series into groups of $(k+1)$ members each. Consider now the last member of each group; call it the terminal member. The wave lengths of the terminal members are

$$
\frac{2 l}{(k+1)}, \frac{2 l}{2(k+1)}, \ldots \frac{2 l}{s(k+1)}, \ldots
$$

These terminal members will appear with normal periods among the free oscillations on the periodically loaded loop. To show this, consider the components of $y_{\mathrm{m}}$ of type $\eta_{\mathrm{s}(\mathrm{k}+1)}$, where 
$s$ may be any integer between 1 and $\infty$. Since in the case of these components $\psi=s \pi$ the period equation becomes:

$$
\frac{h}{\mu} \sin \frac{\mu l}{k+1}+2 \cos \frac{\mu l}{k+1}=2 \cos s \pi .
$$

This equation will be satisfied by putting

$$
\mu=\frac{(k+1) s \pi}{l} .
$$

The free periods of components of type $\eta_{8(\mathrm{k}+1)}$ are now easily obtained.

$$
\begin{gathered}
\nu_{\mathrm{s}}^{2} L C+\nu_{\mathrm{s}} R C=-\mu^{2}=-\frac{(k+1)^{2} s^{2} \pi^{2}}{l^{2}} \\
\therefore \quad \nu_{\mathrm{s}}=-\frac{R}{2 L} \pm i \sqrt{\frac{1}{L C} \frac{(k+1)^{2} s^{2} \pi^{2}}{l^{2}}-\frac{R^{2}}{4 L^{2}}} \\
=-\frac{R}{2 L} \pm i p_{\mathrm{s}} .
\end{gathered}
$$

From the expressions for $y_{\mathrm{m}}$ we deduce:

$$
\eta_{\mathrm{s}(\mathrm{k}+1 \mathrm{C}}=B_{\mathrm{s}(\mathrm{k}+1)} \sin \frac{(k+1) s \pi \xi}{l},
$$

where

$$
B_{\mathrm{s}(\mathrm{k}+1)}=B_{\mathrm{s}(\mathrm{k}+1)}^{1} e^{-\frac{R t}{2 L}} \cos \left(p_{\mathrm{s}(\mathrm{k}+1)} t-\varepsilon_{\mathrm{s}(\mathrm{k}+1)}\right) .
$$

The wave length of this component is $2 l / s(\mathrm{k}+1)$.

The wave lengths of the components of type $\eta_{\mathrm{s}(\mathrm{k}+1)}$ are :

$$
\frac{2 l}{k+1}, \frac{2 l}{2(k+1)}, \frac{2 l}{3(k+1)}, \ldots \frac{2 l}{s(k+1)} \cdots
$$

From these wave lengths and their corresponding periods we see that the oscillations of the type $\eta_{s(k+1)}$ are identical with the terminal members of the groups into which the free oscillations on a uniform loop were divided above. The physical reason why these periods have not been disturbed by the introduction 
of reactance points is easily seen. It is because they have their nodes at the reactance points. The other $k$ members of each group have been disturbed by the presence of the reactance points. The character of this disturbance will be now discussed.

\section{b. Waves of Displaced Period.}

From the expression for $y_{\mathrm{m}}$ we deduce, by putting $\xi=0$,

$$
\begin{gathered}
x_{\mathrm{m}}=\sum_{r=1}^{\infty} \frac{A_{\mathrm{r}} \cos (k-m+2) \frac{r \pi}{k+1} \sin \mu_{\mathrm{r}} \frac{l}{k+1}}{\sin \frac{r \pi}{k+1}} \\
=\sum_{r=1}^{\infty} \xi_{\mathrm{m}, \mathrm{r}}
\end{gathered}
$$

First, consider the wave lengths of these oscillations of type $\xi_{\mathrm{m} . \mathrm{r}}$. Begin with the fundamental

$$
\xi_{\mathrm{m}, 1}=\frac{A_{1} \cos (k-m+2) \frac{\pi}{k+1} \sin \mu_{1} \frac{l}{k+1}}{\sin \frac{\pi}{k+1}}
$$

As $m$ increases from 1 to $\mathrm{k}+2$ the angle $(\mathrm{k}-m+2) \pi / \mathrm{k}+1$ diminishes from $\pi$ to 0 . In Fig. 4 the line $L_{1} L_{1}$ represents the length of the loop. $L_{\mathrm{k}+2}$ is the middle point of it. Points $1,2,3 \ldots k+2$ mark the position of the reactance points. At these points measure off abscissæ equal to $\xi_{1,1} \xi_{2,1} \xi_{3,1} \ldots$ The extremities of these abscissæ will be on the harmonic curve 1 . This curve represents the fundamental oscillation of displaced period. Its wave length is $2 l$, the same as the wave length of the fundamental oscillation before the introduction of the reactance points.

The next component is

$$
\xi_{\mathrm{m}, 2}=\frac{A_{2} \cos (k-m+2) \frac{2 \pi}{\mathrm{k}+1} \sin \mu_{2} \frac{l}{k+1}}{\sin \frac{2 \pi}{k+1}}
$$


As $m$ increases from 1 to $\mathrm{k}+2$ the angle $(\mathrm{k}-m+2) 2 \pi / \mathrm{k}+1$ diminishes from $2 \pi$ to zero. It will, therefore, have four maxima and four zero points on the loop. Its wave length is $l$. This component is represented by curve II in Fig. 4.

Take now the last component but one of the first group of free oscillations of displaced period. It is

$$
\xi_{\mathrm{m}, \mathrm{k}}=\frac{A_{\mathrm{k}} \cos (k-m+2) \frac{k \pi}{k+1} \sin \frac{\mu_{\mathrm{k}} l}{k+1}}{\sin \frac{k \pi}{h+1}}
$$

As $m$ increases from 1 to $k+2$ the angle $(k-m+2) k \pi / k+1$ diminishes from $\mathrm{k} \pi$ to zero. This component has, therefore,

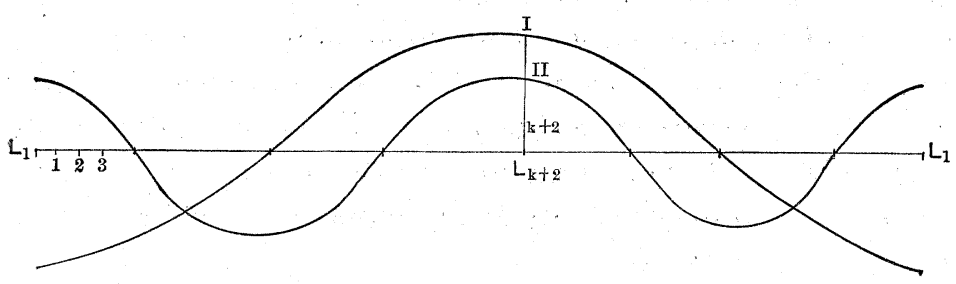

Fig. 4.

$2 \mathrm{k}$ maxima and $2 \mathrm{k}$ zero points on the loop. Its average wave length is $2 l / \mathrm{k}$. The last component of this group is the same as the lowest oscillation of normal period-that is, $\eta_{\mathrm{k}+1}$.

The components of the first group have, therefore, the same average wave lengths as the components of the first group of free oscillations on the loop before the introduction of the reactance points.

It can now be easily shown that this is true for the components of all the other groups, and attention should be called here to the fact that the wave lengths of the free oscillations of displaced period can be considerably smaller than the distance between two consecutive reactance points. The introduction of the reactance points changes, therefore, neither the number of free oscillations nor their average wave length. Their periods, however, and their damping constants will be changed, as will be shown presently. 


\section{Displacement of Periods.}

The periods and damping constants of the oscillations of displaced period are determined from the period equation

$$
\frac{h_{\mathrm{r}} \sin \frac{\mu_{\mathrm{r}} l}{k+1}}{\mu_{\mathrm{r}}}+2 \cos \frac{\mu_{\mathrm{r}} l}{k+1}=2 \cos \frac{r \pi}{k+1} .
$$

The unknown quantity $\nu_{\mathrm{r}}$ contained in $h_{\mathrm{r}}$ and $\mu_{\mathrm{r}}$ is, of course, a complex quantity-that is. we can write

$$
\nu_{\mathrm{r}}=n_{\mathrm{r}}+i p_{\mathbf{r}}
$$

I am not as yet quite ready to discuss fully the general properties of the roots of the period equation. Besides, such a discussion would go considerably beyond the limits of this paper, the principal object of which is to determine the conditions under which the non-uniform conductor of Fig. 3 is equivalent to a uniform loop. I shall limit myself, therefore, to a brief statement of those properties only of this equation which bear directly upon these conditions.

First, since

$$
\cos \frac{r \pi}{k+1}=\cos \frac{[2 s(k+1) \pm r] \pi}{k-\vdash 1}
$$

it follows that the periods and the damping constants of the wave lengths

$\frac{2 l}{r}, \frac{2 l}{2(k+1) \pm r}, \frac{2 l}{4(k+1) \pm r}, \cdots \frac{2 l}{2 s(k+1) \pm r}, \cdots$

are determined from one and the same angle $\psi=r \pi /(\mathrm{k}+1)$. This relation between these wave lengths is illustrated graphically in Table I. The horizontal top bar marked $1,2, \ldots . . \mathrm{k}+1, \ldots$ $2 \mathrm{k}+1,2 \mathrm{k}+2,2 \mathrm{k}+3 \ldots \ldots$ contains a numerical record of all the possible wave lengths

$$
\frac{2 l}{1}, \frac{2 l}{2}, \ldots \frac{2 l}{k+1}, \ldots \frac{2 l}{2 k+1}, \frac{2 l}{2 k+2}, \frac{2 l}{2 k+3} \ldots \ldots
$$

The first vertical column marked $0,1,2,3, \ldots \ldots \mathrm{k}+1 \ldots \ldots$ 
contains the record of all possible values of $r$. The meaning of the black dots is now easily explained. Take, for instance, the row of black dots in the horizontal bar 3. These dots are in columns $3,2 \mathrm{k}-1,2 \mathrm{k}+5,4 \mathrm{k}+1,4 \mathrm{k}+7, \ldots$. This means that if in the period equation

$$
\frac{h}{\mu} \sin \frac{\mu l}{k+1}+2 \cos \frac{\mu l}{k+1}=2 \cos \frac{r \pi}{k+1},
$$

we put $r=3$ then the roots of the equation will give us the periods and the damping constants for the wave lengths

$$
\frac{2 l}{3}, \frac{2 l}{2 k-1}, \frac{2 l}{2 k+5}, \ldots \ldots \ldots
$$

TABLE I.

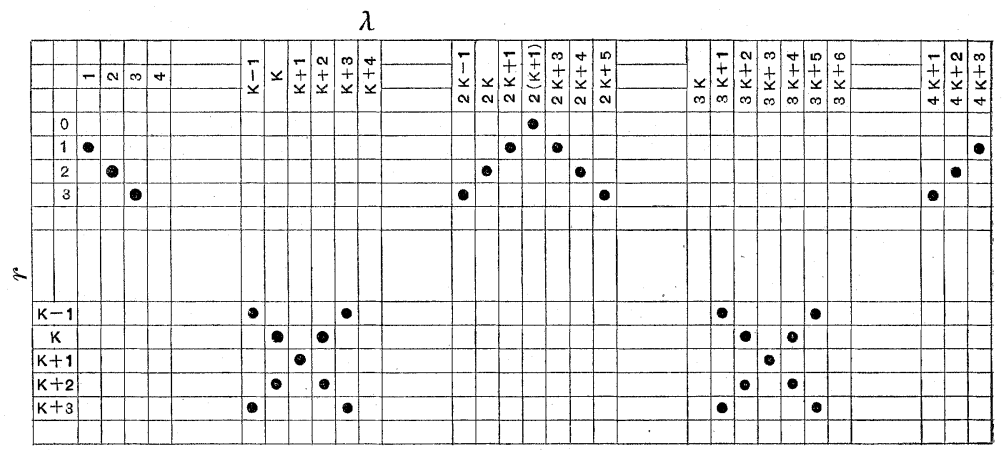

For this reason these may be called the concomitant wave lengths. An inspection of this table shows that the concomitant wave lengths are symmetrically arranged with respect to the wave lengths of normal periods

$$
\frac{2 l}{k+1}, \frac{2 l}{2(k+1)}, \frac{2 l}{4(k+1)}, \ldots \ldots
$$

Of a given series of concomitant wave lengths there is one in each of the groups mentioned above. The first one of these groups contains the longest wave lengths; it may be called the fundamental group. The wave lengths of this group, together with their concomitant wave lengths, give all the possible wave lengths. If in the period equation $r$ is given successively the 
values $0,1,2, \ldots \mathrm{k}+1$, the roots of the resulting $\mathrm{k}+2$ equations. will give the periods and damping constants not only for the wave lengths of the fundamental group, but also their concomitants-that is, for all the wave lengths. It should be observed that the more nearly $\cos r \pi /(\mathrm{k}+1)$ approaches the value +1 or -1 the more closely will the concomitant wavelengths approach the wave lengths of normal period, around which they are symmetrically grouped, and therefore the more closely will their periods approach the period of the central wave length. For instance, the wave lengths

$$
\frac{2 l}{2 k+1} \text { and } \frac{2 l}{2 k+3}, \frac{2 l}{4 k+3} \text { and } \frac{2 l}{4 k+5} \ldots \ldots
$$

are concomitants of wave lengths $2 l / 1$. Their periods are obtained from the period equation by putting $r=1$. This makes $\cos r \pi /(\mathrm{k}+1)$ approach the value +1 , and therefore the periods of wave lengths

$$
\frac{2 l}{2 k+1} \text { and } \frac{2 l}{2 k+3}, \frac{2 l}{4 k+3} \text { and } \frac{2 l}{4 k+5}, \ldots \ldots
$$

will approach the periods of the wave lengths

$$
\frac{2 l}{2(k+1)}, \frac{2 l}{4(k+1)}, \ldots \ldots \ldots
$$

which have been calculated above. When, therefore, $\mathrm{k}$ is large then the roots of the period equation

$$
\frac{h}{\mu} \sin \frac{\mu l}{k+1}+2 \cos \frac{\mu l}{k+1}=2 \cos \frac{r \pi}{k+1},
$$

come out in pairs of nearly equal magnitude for all values of $r$, which are small in comparison to $\mathrm{k}$. This peculiar property of the roots of the period equation suggests a striking resemblance between the oscillations of a periodically loaded conductor and the luminous vibrations of an incandescent gaseous substance, which, in my opinion, deserves serious attention. I expect to discuss this matter more fully at some future occasion. 
Equivalence of a Non-Uniform Conductor of the Second Type to its Corresponding Uniform Condudtor.

Suppose that we now increase the number of reactance points without increasing the total reactance and resistance introduced into the line. In the expression

$$
h=C\left(\nu^{2} \rho+\nu R\right)
$$

put

$$
\rho=\frac{l \lambda^{1}}{k+1}, \quad R=\frac{l R^{1}}{k+1}
$$

Hence,

$$
h=\frac{l}{k+1} C\left(\nu^{2} \lambda^{1}+\nu R^{1}\right)=\frac{l}{k+1} h^{1}
$$

As the number of reactance points is increased $\lambda^{1}$ and $R^{1}$ are to be kept constant; hence, $\rho$ and $R$ must vary inversely as $\mathrm{k}+1$. When $\mathrm{k}$ becomes infinite then all the wave lengths different from zero will be in the tirst group of Table I between the columns 1 and $k+1$. The other columns of this table lose their physical meaning. To show what becomes of the period equation when $\mathrm{k}$ approaches the limit $\infty$ write the period equation in the following form :

$$
\frac{\frac{l}{k+1} h_{1} \sin \frac{\mu l}{k+1}}{\mu}-4 \sin ^{2} \frac{1}{2} \frac{l \mu}{k+1}=-4 \sin ^{2} \frac{1}{2} \frac{r \pi}{k+1}
$$

Since wave lengths different from zero are to be considered, finite values only of $r$ need be considered. Hence, when $\mathrm{k}=\infty$ the period equation becomes

$$
l^{2}\left(h^{1}-\mu^{2}\right)=-r^{2} \pi^{2}
$$

or

$$
C\left[\nu^{2} \lambda^{1}+\nu R^{1}+\nu^{2} L+\nu R\right]=-\frac{r^{2} \pi^{2}}{l^{2}},
$$

or

$$
C\left[\nu^{2}\left(\lambda^{1}+L\right)+\nu\left(R^{1}+R\right)\right]=-\frac{r^{2} \pi^{2}}{l^{2}} .
$$


This equation is, as it ought to be, the period equation of a uniform conductor of inductance $\lambda^{1}+L$, resistance $R^{1}+R$, capacity $C$ per unit length. This conductor is the so-called corresponding uniform conductor of the periodically loaded loop.

When $\mathrm{k}$ is large but not infinite the same relation will exist approximately, and it is proposed to ascertain now the degree of this approximation.

We have seen that $l \mu /(\mathrm{k}+1)$ varies with $r$ as follows:

$$
\frac{l \mu}{k+1}=s \pi, \quad \text { where } r=s(k+1)
$$

$s$ being any integer from 0 to $\infty$. Thus, when

$$
\begin{aligned}
& \frac{r \pi}{k+1} \text { is } 0, \pi, 2 \pi, 3 \pi, 4 \pi, \ldots \ldots \ldots . \\
& \frac{\mu l}{k+1} \text { will be } 0, \pi, 2 \pi, 3 \pi, 4 \pi \ldots \ldots \ldots
\end{aligned}
$$

Since $\mu l /(\mathrm{k}+1)$ varies continuously with $r$ it follows that when $r \pi /(\mathrm{k}+1)$ is small $\mu l /(\mathrm{k}+1)$ will also be small, since both vanish simultaneously. It is well to introduce here the physical meaning of $r /(\mathrm{k}+1)$. Let $\lambda_{\mathrm{r}}$ be the wave length of the wave for which $\psi=\frac{1}{2} r \pi /(\mathrm{k}+1)$, then

$$
\lambda_{\mathrm{r}}=\frac{2 l}{r}, \text { or } r=\frac{2 l}{\lambda_{\mathrm{r}}}
$$

Therefore,

$$
\frac{r \pi}{k+1}=\frac{2 \pi}{\lambda_{\mathrm{r}}} \frac{l}{k+1}=2 \pi \frac{l_{1}}{\lambda_{\mathrm{r}}}
$$

where $l_{1}$ is the distance between two consecutive coils. Let $2 \pi$ be the angular distance of $\lambda_{\mathrm{r}}$ and $\theta_{\mathrm{r}}$ be the angular distance of $l_{\mathrm{L}}$ corresponding to the period of $\lambda_{\mathrm{r}}$, then

$$
\frac{l_{1}}{\lambda_{\mathrm{r}}}=\frac{\theta_{\mathrm{r}}}{2 \pi}
$$

Therefore,

$$
\frac{r \pi}{k+1}=\theta_{r}
$$


Since for small values of $r \pi /(\mathrm{k}+1)$ the angle $\mu l /(\mathrm{k}+1)$ is nearly equal to $r \pi /(\mathrm{k}+1)$ it follows that within these limits $\mu l /(\mathrm{k}+1)$ is approximately equal to the angle $\theta_{\mathrm{r}}$. It follows, therefore, that wave lengths for which $\theta_{\mathrm{r}}$ is sufficiently small the period equation

$$
\frac{l}{k+1} \frac{h^{1}}{\mu} \sin \frac{\mu l}{k+1}-4 \sin ^{2} \frac{1}{2} \frac{l \mu}{k+1}=-4 \sin ^{2} \frac{1}{2} \frac{r \mu}{k+1}
$$

can be written

$$
\left(\frac{l}{k+1}\right) h^{1}-\left(\frac{l}{k+1}\right)^{2} \mu^{2}=-\left(\frac{r \pi}{k+1}\right)^{2}
$$

or

$$
C\left[\nu_{\mathrm{r}}^{2}\left(\lambda^{1}+L\right)+\nu_{\mathrm{r}}\left(R^{1}+R\right)\right]=-\left(\frac{r \pi}{k+1}\right)^{2},
$$

that is, the periodically loaded conductor has approximately the same period, damping constant and, of course, the same wavelength as its corresponding uniform conductor. The degree of approximation is of the same order as the degree of approximition between one-half of the angular distance separating tuo consecutive coils and the sine of that distance.

It should be observed that if the whole loop is divided into a number of equal parts and the consecutive parts are connected to each other by mutual induction, then the non-uniform conductor thus obtained will act in the same way as the non-uniform conductor of Fig. 3 of this section. Another arrangement, which is equivalent to the two arrangements so far described, is obtained by placing bridges at periodic intervals of the uniform loop, each bridge consisting of a coil of proper resistance and inductance, and the distance between the bridges being adjusted in accordance with the general rule formulated here. The mathematical analysis of these two arrangements does not differ essentially from the one given here, and can be easily worked out.

A rule governing the degree of approximation between a nonuniform conductor and its corresponding uniform conductor has thus been established, and it has been proved that the same rule is applicable to both types of non-uniform conductors, and also to both forced and free oscillations. The principal object of this investigation has, therefore, been accomplished. 


\section{Drscussion.}

The President:- Seven years ago the electrical fraternity was startled with a paper on Ocean Telephony. But seven years have passed and we have not yet telephoned across the ocean. The present paper differs from this other one, which was read by an Englishman, in that, in the first place it makes no such extravagant claims as ocean telephony, and in the second place it verifies by actual experiment, the application of a very interesting theory. Papers like this, and the preceding one, show what good results can be accomplished by a careful and intelligent application of correct theory. Such papers reflect great credit on the Institute. The paper is now open for discussion.

Mr. Steinmetz:-Mr. President, I have been very much pleased to listen to this highly interesting and extremely important paper, which marks a decided advance in the art of telephony, as I believe. Now, coming first to a comparison with previous propositions for improving the method of telephony over cables, there is an essential difference between the present case and the previous ones. In the latter, if I remember rightly, it was proposed to distribute inductances along the cable, these inductances to be shunted across the cable. The capacity of the cable and the static capacity act as a shunt across the line. If you want to compensate for the effect of capacity by self-induction, or self-induction by capacity, one must be in shunt and the other in series. That is to make up for the electrostatic capacity of the cable. That is the case here. The phase relation and frequency will remain the same throughout the whole line. This was not the case with the previous proposition. The problem of transmitting alternate currents leads to two very distinct phenomena and problems, that of transmitting very large powers at very low frequency relatively, and high voltages, with the so-called electric power appliances, and then the transmitting of very small powers at very high frequencies at very low voltage. That is telephony. The theoretical aspect of the problem is the same, but they have to be conducted entirely different. The solution of the problem in the one case is entirely inapplicable to the other case. Theory has to go ahead of investigation, except by mere luck in an individual case you find a solution, and in the next case you reach the same solution and it is entirely wrong. We have found means to compensate for the effect of self-induction by synchronous apparatus. Self-induction is not an objection any wore; it is rather an advantage. We have a similar problem in he system of telephony, only there it is not the self-induction which is very small in the ordinary telephone, at the frequencies and voltages in current use, but it is the capacity which is the dangerous objection. The attempt to nse the solution which is satisfactory in power transmission would not be fair because the two conditions are entirely different. The shunt across is en- 
tirely unsatisfactory and makes the problem worse, it spoils what little transmission of speech we can get. In America we have investigated the transmission at very high voltages and at relatively low frequencies. There is a certain value in self-induction but it is not at all essential in most cases to have exactly this value. You can get half as much and still the transmission is very much improved over what it would be without the self-induction. The more you put the self-induction in the better it becomes.

Referring to the second part of the paper, the mathematical side, I have not read it yet, so I cannot say anything about it. If any further suggestions occur to me 1 shall cornmunicate them in writing.

Dr. Crenore :-Mr. President, I would like to make one inquiry. It occurs to me that in looking up the proportions to form a distortionless circuit the value of $i$ or the reduction required is invariable; that if you were to send a sine wave into the cable the energy that you get through is necessarily very much reduced from what it would be without the induction. I would like to ask Dr. Pupin for a comparison of the amount of energy that he gets through this 250 -mile cable by sending a constant voltage, say of 30 to 50 volts. I would like to form a comparison between the amount you get through the cable when you have those cuils in, and when you do not, because it seems to me very important as to the distance that you will be able to cover.

Dr. Pupin :-The ratio is 1 to 10,000 .

Dr. Crehore:-The ratio between the energy you would receive and otherwise?

Dr. PuPIN :-Yes. By making a certain arrangement you can get it constant within practical limits. In this particular case the ratio of the current put in at one end and the current received at the other end is 50 to 1 . Two per cent. of the current that is put in at one end is received at the other end when you put the coils in, and of course that is all that is needed for telephony over that length. If you did not have the coils in, instead of 1 to 50 you would have 1 to 125,000 .

Dr. OreHORE:-You mean you would get a larger amount with the coils in?

DR. PUPIN :--Y Yes; very much larger. I say 1 to 125,000 . I could not get any current through at the other end at all that I could detect with the telephone. I believe you do not get anything through to speak of.

DR. CREHORE :- Sending a simple sine wave?

Dr. PuPIN :- Sending a simple sine wave by means of the alternator; that is, using about 15 volts at one end. This calculation refers to about 625 periods per second.

Prof. Franklin:- What is the condition of this circuit with regard to the distortion? 
Dr. Pupin:- The attenuation constant is the same for all frequencies, and therefore the circuit is distortionless. If there was any difference at all in the relation between the attenuation and the frequency I did not notice it.

Prof. Franklin:--You did not take precaution beforehand to satisfy the conditions.

Dr. Pupin :-Yes. I did not want any distortion. I could have detected it experimentally up to about two per cent. on an average. $\mathrm{Up}$ to that limit there is no dependence between the frequency and the attenuation. If it is anything less than two per cent. it does no harm.

Mr. Steinmetz:- Since they have been speaking of distortion, I desire to sound a note of warning. As I said before, the solution in telephony is entirely different from that in power transmission at low frequency. That does not mean that the proper method of alternating current transmission is to put in as much capacity as to make it distortionless. Take a telephone cable and impress it with the ordinary electro-motive force; then you will require a very large impressed power to get the same current out of the cable without inductance.

Dr. Pupin :-I would like to correct a statement which our worthy president has made. It is not an error on his part, but, rather a misunderstanding. He said I did not make a claim to telephony on an ocean cable. I do decidedly make that claim.

The President :-You did not make the claim in the paper.

Dr. Pupin :-I did not make it in this paper, because I reserved it for another paper which I expect to bring very soon before this Institute, in which I shall discuss the cable problem in all its details. But I do now state decidedly that I am convinced that telephoning over a cable of reasonable length, say 1,000 miles to start with, is quite possible and nothing at all extraordinary.

Prof. Franklin :-I would like to ask Dr. Pupin a question, and that is, why not apply this method which you have been working upon to simply increasing the possible speed of telegraphy? It applies to that equally with telephony. Why telephone across? Why not apply it to increasing the speed of signaling.

Dr. PUPIN :-That goes without saying. It will help telegraphy as well as telephony. Of course it would increase the speed of telegraphy. You can take a distortionless loaded conductor like the one described in this paper and transmit five or ten different messages over the same wire. That would be even more important than telephony, but I don't know that it would be as interesting from a scientific point of view.

The President:-When I said that Dr. Pupin did not make any claim to ocean telephony I meant to compliment him; I ineant to say that he claimed no more than what he had really accomplished, and that it was in this that his paper differed very radically from the other one to which I referred. Concerning 
ocean telegraphy, or at least trans-A tlantic telegraphy, it should not be forgotten that in telephoning east and west there is another factor which enters, and that is that the business days in London and New York, for instance, differ by five hours and do not overlap for a sufficient length of time to make it pay to have a cable for telephoning across the Atlantic unless such a cable could also be used at other times for telegraphing. To simply have a cable for telephony I am afraid would hardly pay, because the ti ne of utilization would only be about two or three hours out of the 24 . This does not apply to telephoning north and south.

Dr. Crenore :-I would like to inquire whether I am anticipating too much to ask Dr. Pupin whether he is prepared to show a form of cable which he could put such coils into or indicate how a cable is to be constructed for submarine work, or whether he chooses to reserve that for the paper which he intends to present in the future?

Dr. PuPIn:-No; it is no secret. I did not talk about it, because I have not yet sufficient experimental data on that particular form of cable, but it is a cable of the same kind as the one discussed in this paper. There is only this distinction, and it is only a distinction in degree and not in principle at all. I said a little while ago that on a land line of 2,000 miles, say between here and New Orleans, if you wanted to introduce coils at a distance of two miles it would be sufficient, but if you have a cable where the capacity is large, then the coils have to be placed at much smaller distances apart. I made a calculation for a particular form of cable, any three-tenths of a microfarad per mile and four ohms resistance per mile, and in order to give the cable a sufficiently small attenuation constant for 750 periods I introduced inductances so as to give three-tenths of a henry per mile. I made the reactance coils smaller. I wound the core two inches and a half external and one itch internal diameter and six inches long with very fine iron wire. That is where the whole trick comes in-to have fine wire. It is necessary to have iron wire two mils in diameter. I am informed by some manufacturers of iron wire in Worcester, Massachusetts, that they would fill such an order if I wanted it at once. This is very tine Swedish iron wire. They can draw it very easily down to two mils diameter. You wind two coils in inductive relation to each other, each 40 turns, copper wire, and then you place them eight coils per mile. That is at a distance of between 600 and 700 feet apart. Coils like that can go right into the sheathing of the cable. It is all a question of Foucault current losses in the iron. The Foucault current losses can be reduced to a very small amount. I calculate that 25 coils like that, with a frequency of 750 periods, would be equivalent to adding one ohm to the line. So that form of cable is a practicable form. Instead of having them 700 or 800 feet you can make them a little more frequent.

Mr. Hoмmes:-It appears to me that the main point just at 
present is to see whether it is practicable to telephone for any such distance through the eapacity of an ocean cable and resistance, and if it is, the working out would be a matter of detail. Even if the coile could not be made in just this manner, it appears to me it would not matter. If they were a little larger there could be some mechanical arrangement devised so as to take the strain off the coils, and while this arrangement as well as the other would undoubtedly make the cost of manufacturing the cable higher, probably the income from being able to telephone across would warrant the expense; and it appears to me the main point would be to find out whether it is practicable to telephone any great distance over an ocean cable by means of self-induction added at intervals as shown.

Dr. Pupin:-But one more remark. Nobody cares so much for ocean telephony anyhow. It is not so very important. It is very interesting from a purely scientific point of view, but whether it is of so very great commercial importance I am not prepared to say. I am rather inclined to think the other way. I think telephony on land lines is the point. We have reached the limit of telephony over land lines. We cannot telephone over 1,000 or 1,200 miles with any sort of satisfaction. The telephone men themselves tell me that it is a somewhat unsatisfactory business to telephone between St. Louis and New York. You have got to repeat your sentences very often. The lines are too expensive. The circuit between New York and Chicago costs for copper alone $\$ 250,000$. Now, supposing you wanted to extend the limit of telephony and wanted to go to San Francisco or to Kansas City, unless you spend four or five times as much per mile on the circuit you cannot telephone at all. Then you have other considerations-the weight of the wire, etc.--and according to the views of the telephone men themselves it is impracticable to carry on the telephone business over a distance longer than 1,200 miles. By spending a few dollars for these coils, at proper distances apart, I would undertake to build a line between New York and New Orleans, although I am not a practical engineer and I do not care so very much for commercial work, but just for the sake of showing what could be done I would undertake to build a line between New York and New Urleans that would be four ohms per mile; that is twice the resistance per mile of the standard wire between New York and Chicago. I think that this is the really important problem-the problem of extending the limit of telephony over land lines.

The President :-If there is no further discussion we will go on with the next paper. The title of the next paper and the last one is "Notes on Synchronous Converters," by Prof. R. B. Owens, of Montreal.

[This paper will be printed subsequently.-EDrTor.] 